\title{
PANCHROMATIC HUBBLE ANDROMEDA TREASURY. IX. A PHOTOMETRIC SURVEY OF PLANETARY NEBULAE IN M31
}

\author{
Mark J. Veyette ${ }^{1,2}$, Benjamin F. Williams ${ }^{1}$, Julianne J. Dalcanton ${ }^{1}$, Bruce Balick ${ }^{1}$, Nelson Caldwell ${ }^{3}$, \\ Morgan Fouesneau $^{1,4}$, Léo Girardi ${ }^{5}$, Karl D. Gordon ${ }^{6,7}$, Jason Kalirai $^{6,8}$, Philip Rosenfield $^{9}$, and Anil C. Seth ${ }^{10}$ \\ ${ }^{1}$ Department of Astronomy, University of Washington, P.O. Box 351580, Seattle, WA 98195, USA; mveyette@uw.edu \\ ${ }^{2}$ Astronomy Department, Boston University, 725 Commonwealth Avenue, Boston, MA 02215, USA \\ ${ }^{3}$ Harvard-Smithsonian Center for Astrophysics, 60 Garden Street, Cambridge, MA 02138, USA \\ ${ }^{4}$ Max-Planck-Institut fur Astronomie, Königstuhl 17, D-69117 Heidelberg, Germany \\ ${ }^{5}$ Osservatorio Astronomico di Padova-INAF, Vicolo dell'Osservatorio 5, I-35122 Padova, Italy \\ ${ }^{6}$ Space Telescope Science Institute, 3700 San Martin Drive, Baltimore, MD 21218, USA \\ ${ }^{7}$ Sterrenkundig Observatorium, Universiteit Gent, B-9000 Gent, Belgium \\ ${ }^{8}$ Center for Astrophysical Sciences, Johns Hopkins University, Baltimore, MD 21218, USA \\ ${ }^{9}$ Department of Physics and Astronomy G. Galilei, University of Padova, Vicolo dell'Osservatorio 3, I-35122 Padova, Italy \\ ${ }^{10}$ Department of Physics and Astronomy, University of Utah, Salt Lake City, UT 84112, USA \\ Received 2014 April 7; accepted 2014 July 15; published 2014 August 22
}

\begin{abstract}
We search the Hubble Space Telescope (HST) Advanced Camera for Surveys and Wide Field Camera 3 broadband imaging data from the Panchromatic Hubble Andromeda Treasury (PHAT) survey to identify detections of cataloged planetary nebulae (PNs). Of the 711 PNs currently in the literature within the PHAT footprint, we find 467 detected in the broadband. For these 467, we are able to refine their astrometric accuracy from $\sim 00^{\prime} 3$ to $0{ }^{\prime} .05$. Using the resolution of the $H S T$, we are able to show that 152 objects currently in the catalogs are definitively not PNs, and we show that 32 objects thought to be extended in ground-based images are actually point-like and therefore good PN candidates. We also find one PN candidate that is marginally resolved. If this is a PN, it is up to 0.7 pc in diameter. With our new photometric data, we develop a method of measuring the level of excitation in individual PNs by comparing broadband and narrowband imaging and describe the effects of excitation on a PN's photometric signature. Using the photometric properties of the known PNs in the PHAT catalogs, we search for more PNs, but do not find any new candidates, suggesting that ground-based emission-line surveys are complete in the PHAT footprint to $\mathrm{F} 475 \mathrm{~W} \simeq 24$.
\end{abstract}

Key words: galaxies: individual (M31) - planetary nebulae: general

Online-only material: color figures, machine-readable tables

\section{INTRODUCTION}

As the nearest large spiral galaxy, M31 offers a unique opportunity to study planetary nebulae (PNs) on a galactic scale. Stars born with masses between $0.8-8 M_{\odot}$ will likely go through a PN phase, making PNs good tracers of old stellar populations. Due to the short timescales between the asymptotic giant branch (AGB) and PN phases, the distribution and kinematics of PNs are expected to be identical to their parent population. Studying PNs as a population provides insight into galactic structure and evolution. Understanding their evolution individually can provide insight into PN structure, formation, and enrichment of the interstellar medium and provide constraints for future PN evolutionary models. Studying PNs has the additional benefit of ease of detection and classification due to their strong emission features and unique spectra. Furthermore, the population of PNs in M31 all lie at the same distance, making it possible to directly relate the luminosity and emission of a large and diverse sample of PNs.

Previous surveys have identified and studied thousands of PNs throughout the disk and bulge of M31 (Ciardullo et al. 1989; Hurley-Keller et al. 2004; Halliday et al. 2006; Merrett et al. 2006). Recent spectroscopic studies have begun to dig deeper into their physical properties (Kwitter et al. 2012; Sanders et al. 2012). The largest survey of M31 PNs to date is a deep kinematic survey performed by (Merrett et al. 2006, hereafter M06) using the purpose-built Planetary Nebula Spectrograph (Douglas et al.
2002), which can simultaneously detect PNs and measure their radial velocities. M06 identified 3300 emission-line objects in M31, of which 2615 are likely to be PNs.

Single-purpose spectroscopic surveys are very useful for studying the properties of individual PNs but can be time consuming and costly. In contrast, multi-purpose photometric surveys can offer substantial, albeit less detailed information about previously discovered PNs without the need for additional observations and can even be used to identify previously undiscovered PNs (Kniazev et al. 2014). Broad spectral coverage allows one to probe large spans of the spectral energy distributions (SEDs) of PNs and can be used in concert with spectroscopic surveys to calibrate photometric data for the study of PN emission. Although this photometric information alone is not as detailed as spectroscopic data, it is sufficient to place broad constraints on the properties of the overall strength of the PN's emission lines and underlying stellar continuum for much larger populations than are accessible spectroscopically.

PNs are typically identified by searching for objects with bright [O III] $\lambda 5007$ lines. Traditional large-area surveys use the difference of on- and off-band narrowband photometry to select PN candidates, which are then followed up spectroscopically to exclude $\mathrm{H}$ II regions and Wolf-Rayet (WR) stars, which also emit strong [O III] lines. Ground-based spectroscopy is limited to only the brightest PNs and restricted by atmospheric windows. For the M06 survey, spectroscopic follow up was replaced by cuts of extended objects and 
objects with low $F_{5007} / F_{\mathrm{H} \alpha}$ ratios measured from narrowband imaging from the Local Group Survey (LGS; Massey et al. 2006). While this method provided a deeper and larger survey without the need for spectroscopic follow up, it is also subject to more contamination. High-resolution imaging provides a cleaner view of the sources of the [O III] emission, improving rejection of non-PN sources, particularly extended $\mathrm{H}$ II regions which are the dominant contaminating object in PN surveys.

In Section 2 of this paper, we describe how we identified PNs in the Panchromatic Hubble Andromeda Treasury (PHAT) catalog by cross-identification with M06. In Section 3, we present the PHAT PNs catalog and characterize basic ultraviolet (UV), optical, and near-infrared (NIR) photometric properties of PNs. We discuss our results in Section 4 and summarize them in Section 5.

\section{DATA ACQUISITION AND ANALYSIS}

\subsection{The Panchromatic Hubble Andromeda Treasury}

The Panchromatic Hubble Andromeda Treasury (PHAT) is a Hubble Space Telescope (HST) multi-cycle imaging survey covering roughly one-third of M31's star-forming disk out to $20 \mathrm{kpc}$ (Dalcanton et al. 2012). Observations were made with the HST using the Advanced Camera for Surveys (ACS) and Wide Field Camera 3 (WFC3), with six filters spanning the UV (WFC3/UVIS F275W and F336W), the optical (ACS/ WFC F475W and F814W), and the NIR (WFC3/IR F110W and F160W). ${ }^{11}$ For full details of the data acquisition and analysis, see Dalcanton et al. (2012).

Briefly, photometry was reduced using the DOLPHOT 1.2 (Dolphin 2000) software package which uses iterative point-spread function (PSF)-fitting photometry on neighborsubtracted images to fit the sky and PSF simultaneously. This process is done for each peak in a stack of images across different filters of the same camera. DOLPHOT aligns each image in a stack using hundreds to thousands of bright stars in the field to achieve the precise alignment needed for its photometric processing. Further internal astrometric alignment was performed for each camera. The cameras were then aligned with each other and with a global reference frame. The reference frame was provided by archival $i^{\prime}$ data from MegaCam Canada-France-Hawaii Telescope (CFHT). The error in ACS/WFC to CFHT alignment is $0{ }^{\prime} .05$ and the relative error in alignment across the three cameras is 0.01 . Cross-identification with M06 is therefore limited by the astrometric accuracy of M06-reported to be 0.'34 and 0'.16 in right ascension (R.A.) and declination (decl.), respectively.

We avoided restricting our search to the PHAT point source catalogs. Rather, we cross-identified PNs using the ACS "*.phot" files produced by DOLPHOT. We used these raw DOLPHOT output files, which contain sources that may poorly fit the PSF, so as not to exclude any extended PNs.

PHAT is a multi-release program that is still in progress. This paper covers only the PHAT data available at the time the work for this paper was done. PHAT consists of a total of 23 bricks, each containing $18 H S T$ pointings. This paper covers only bricks $2,4,5,6,8,9,12,14,15,16,17,18,19,21,22$, and 23. Brick 1 was excluded from this paper, though it is currently available, due to heavy crowding effects that made it difficult to identify $\mathrm{PNs}$ in the inner regions of M31.

\footnotetext{
11 All data files are publicly available and described at http://archive.stsci.edu/prepds/phat/.
}

\subsection{Identifying PNs in PHAT}

All PNs analyzed in this paper were originally identified as part of the M06 deep kinematic survey of M31, which covered M31's disk out to a radius of $2^{\circ}(27.4 \mathrm{kpc})$. M06 identified 2615 likely PNs down to a reported completeness of $m 5007 \simeq 23.75$ for the [O III] $\lambda 5007$ line. Of these 2615 , 711 are in the current PHAT footprint. Individual PN candidates have reported uncertainties in their $m 5007$ measurements of $0.07 \mathrm{mag}$ and in their R.A. and decl. measurements of 0.34 and $0{ }^{\prime \prime} 16$, respectively. We cross-identified sources in the M06 catalog with photometric data from PHAT.

\subsubsection{PNs in the PHAT Filters}

Figure 1 shows throughput curves for each of the PHAT filters overlaid on a model PN spectrum. The bandpasses of the two UV filters, F275W and F336W, overlay many weak emission lines together with a significant stellar and nebular continuum (see Bianchi et al. 1997). The majority of nebular emission-line flux falls under the bandpass of the F475W filter, namely the strong emission lines: [O III] $\lambda 5007$, [O III] $\lambda 4959$, $\mathrm{H} \beta$, and $\mathrm{H} \gamma$. Therefore, $\mathrm{F} 475 \mathrm{~W}$ flux is due largely to nebular emission lines. The F814W bandpass overlies a few weak emission lines, though the majority of flux in F814W is likely due to stellar and Paschen continuum. A few weak emission lines also fall under the F110W bandpass, which could have a significant or dominant contribution to the total flux given the weaker underlying stellar continuum in the NIR. Flux in F160W, however, is most likely entirely due to continuum from the central star as there are no emission lines that could have a significant contribution to the total F160W flux. Table 1 lists likely main contributors of PNs flux in each of the PHAT filters.

The vast majority of PN emission-line flux within the PHAT spectral range falls in the F475W bandpass. Thus, most PNs have unusual colors, particularly in three-color images where F475W drives the green channel. Figure 2 shows F814W:F475W:F336W R:G:B images in which PNs appear anomalously blue-green, making them easy to visually differentiate from surrounding stars. We generated equivalent images for all the PHAT data and used them to visually identify PNs, as described in the following section.

\subsubsection{PHAT Astrometry and Photometry}

To assess the relative positional accuracy of the M06 and PHAT catalogs, we performed an initial visual search for anomalously blue-green objects within three times the $1 \sigma$ uncertainties of the M06 reported positions. We found likely optical counterparts as far as $2^{\prime \prime}$ from their cataloged position. We therefore adopted $3^{\prime \prime}$ (11 pc at the distance of M31) as a search radius.

There are, on average, $\sim 600$ objects in PHAT within $3^{\prime \prime}$ of an M06 PN location. We therefore require additional constraints to identify the PHAT counterpart. We first take advantage of the fact that there is a strong linear relation between the quoted M06 $m 5007$ magnitude and the PHAT F475W magnitude, since the majority of F475W flux is due to the $[\mathrm{O}$ III $] \lambda 5007$ line. We show this relation in Figure 3 for the final catalog. A linear fit to this relation finds $\mathrm{F} 475 \mathrm{~W}=-0.2240+1.0187 \times m 5007$. In addition to magnitude, we also considered cross-identifying sources based on optical color, positional offset from M06, and the "sharpness" and "roundness" of the PSF. The most distinguishing identification parameters were optical color and the expected F475W magnitude estimated from the M06 


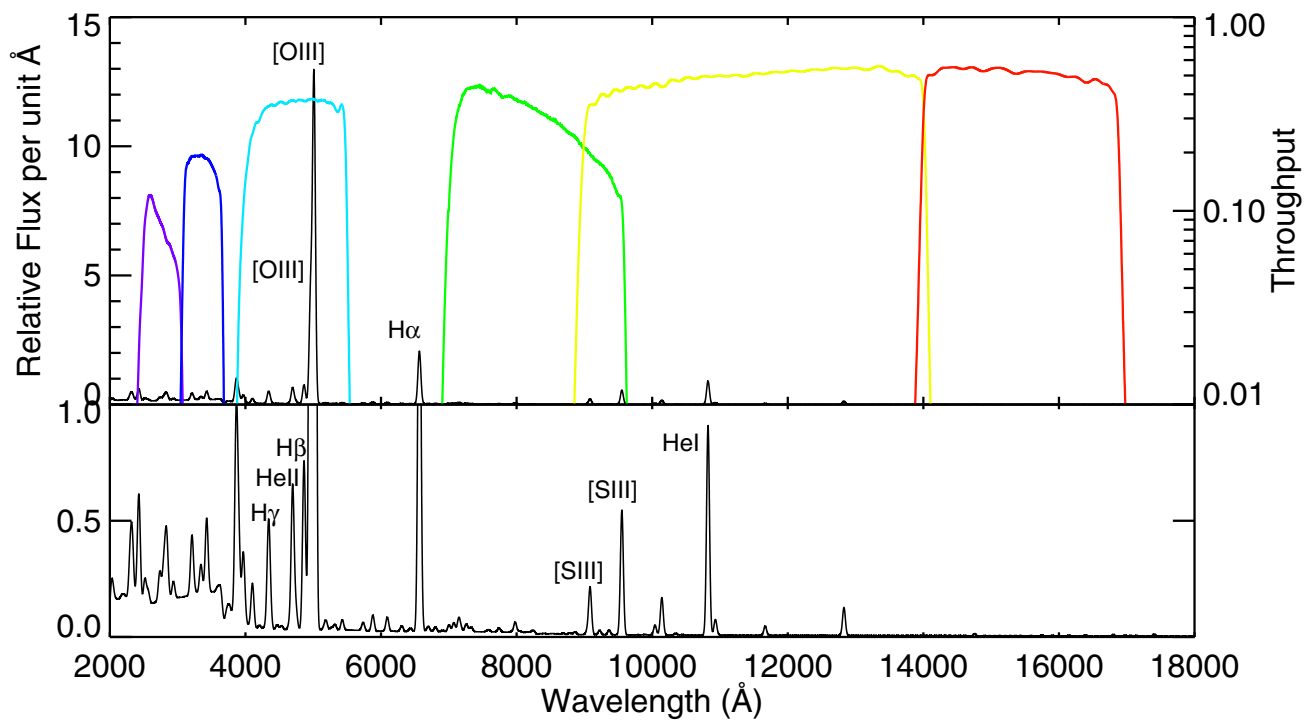

Figure 1. Top: a model spectrum (black line) for a PN model close to its maximum [O III] $\lambda 5007$ luminosity and with high excitation. This particular model was extracted from the Marigo et al. (2001, 2004) database of simplified evolutionary models, in which the photoionization of a spherically symmetric expanding shell is simulated with Ferland et al. (2013) Cloudy code v08.01. Some of the main contributors to the nebular flux are marked in the figure. In addition, there is a significant flux contribution from the stellar and nebular continuum, especially in the UV, as listed in Table 1. Also shown are filter throughput curves (colored lines) for filters: (from left to right) F275W, F336W, F475W, F814W, F110W, and F160W. Bottom: a closer look at the continuum and weaker emission lines.

(A color version of this figure is available in the online journal.)

Table 1

Flux Contribution by Filter

\begin{tabular}{|c|c|}
\hline Filter & Likely Main Flux Contributors \\
\hline F275W & $\begin{array}{l}\text { Stellar continuum, nebular continuum, [Fe IV] } \lambda 2836,[\mathrm{Fe} \text { IV }] \lambda 2829,[\mathrm{Fe} \text { IV }] \lambda 2567,[\mathrm{C} \text { IV }] \lambda 2529, \\
\mathrm{He}_{\text {II }} \lambda 2733, \mathrm{He}_{\mathrm{II}} \lambda 2511, \mathrm{He} \text { II } \lambda 2386,[\mathrm{Ar} \text { IV }] \lambda 2854\end{array}$ \\
\hline F336W & Stellar continuum, nebular continuum, Balmer continuum, He II $\lambda 3203$ \\
\hline F475W & He II $\lambda 4686$, Н I $\lambda 4861$, H I $\lambda 4340,[$ Ar IV] $\lambda 4740,[$ III] $\lambda 5007,[$ O III $] \lambda 4959$ \\
\hline F814W & Stellar continuum, Paschen continuum, $[\mathrm{Cr} v] \lambda 7979, \mathrm{He}$ I $\lambda 7065,[\mathrm{~S} \mathrm{III}] \lambda 9069,[\mathrm{Ar}$ III $] \lambda 7135$ \\
\hline F110W & Stellar continuum, He II $\lambda 10120, \mathrm{He}_{\mathrm{I}} \lambda 10830, \mathrm{H}$ I $\lambda 12820,[\mathrm{~S}$ III $] \lambda 9532,[\mathrm{~S}$ III $] \lambda 9069$ \\
\hline F160W & Stellar continuum, He II $\lambda 4760$ \\
\hline
\end{tabular}

Note. An extensive but incomplete list of likely flux contributors by filter.

m5007 magnitude. The round and sharp PSF values from PHAT's photometry were used mainly to weed out artifacts and spurious objects such as cosmic rays from the uncut "*.phot" files.

To automatically select candidates on the basis of the above parameters, we used an initial training set of visually identified PNs. We calculated the average value and $1 \sigma$ spread of each identification parameter (expected F475W, optical color, positional offset from M06, sharpness, and roundness). For each object in PHAT within 3" of an M06 PN location, the differences between the training set's and the object's parameter values were normalized by the $1 \sigma$ spreads of the parameter values and then averaged over all parameters giving double the weight to the optical color and estimated F475W magnitude. The resulting value, noted as $z_{\mathrm{PN}}$, is the object's standard score of the deviation from typical PNs identified in PHAT. Objects with $z_{\mathrm{PN}}<1$ are highly probable PN candidates. Objects with $z_{\mathrm{PN}}<1.5$ are probable PN candidates. Objects with $z_{\mathrm{PN}}<3$ are possible PN candidates. Equations (1) and (2) define $z_{\mathrm{PN}}$, where for parameter $x, x_{\mathrm{obj}}$ is the object's value, $\bar{x}_{\mathrm{PN}}$ is the training set's average value, and $\sigma_{x_{\mathrm{PN}}}$ is the $1 \sigma$ spread:

$$
z_{x} \equiv \frac{\left|x_{\mathrm{obj}}-\bar{x}_{\mathrm{PN}}\right|}{\sigma_{x_{\mathrm{PN}}}}
$$

$$
\begin{aligned}
z_{\mathrm{PN}} \equiv & \left(2 z_{\mathrm{F} 475 \mathrm{~W}}+2 z_{\text {color }}+z_{\mathrm{RA}}\right. \\
& \left.+z_{\text {Dec }}+z_{\text {sharp }}+z_{\text {round }}\right) / 8
\end{aligned}
$$

We attempted to identify new PNs in M31 using the optical color and F475W magnitude space occupied by the M06 PNs. We looked for [O III] emission in the LGS [O III] images (Massey et al. 2006) to confirm these candidate PNs. None of these candidates had measurable [O III] emission. Therefore, this attempt did not result in the identification of any new PNs, suggesting M06 is complete to the LGS [O III] detection limit. An earlier attempt to photometrically identify new PNs in PHAT was made by selecting sources in the bulge of M31 with F475W $<24.0$, F475W - F814W $<-0.3$, and no matching source in the literature. Spectroscopic follow up of 16 of these candidates found that 7 showed emission lines indicative of PNs. A larger campaign could lead to the discovery of PNs in the inner regions of M31, where previous surveys suffered extreme crowding.

Finally, all catalogs and figures presented contain only photometric measurements with a signal-to-noise ratio $(\mathrm{S} / \mathrm{N})$ greater than four. This $\mathrm{S} / \mathrm{N}$ cut, which is necessary to ensure only accurate, high-quality measurements are analyzed, resulted in the culling of $120 \mathrm{~F} 275 \mathrm{~W}$ measurements, 2 F336W measurements, 1 F814W measurement, and 29 F160W measurements. 

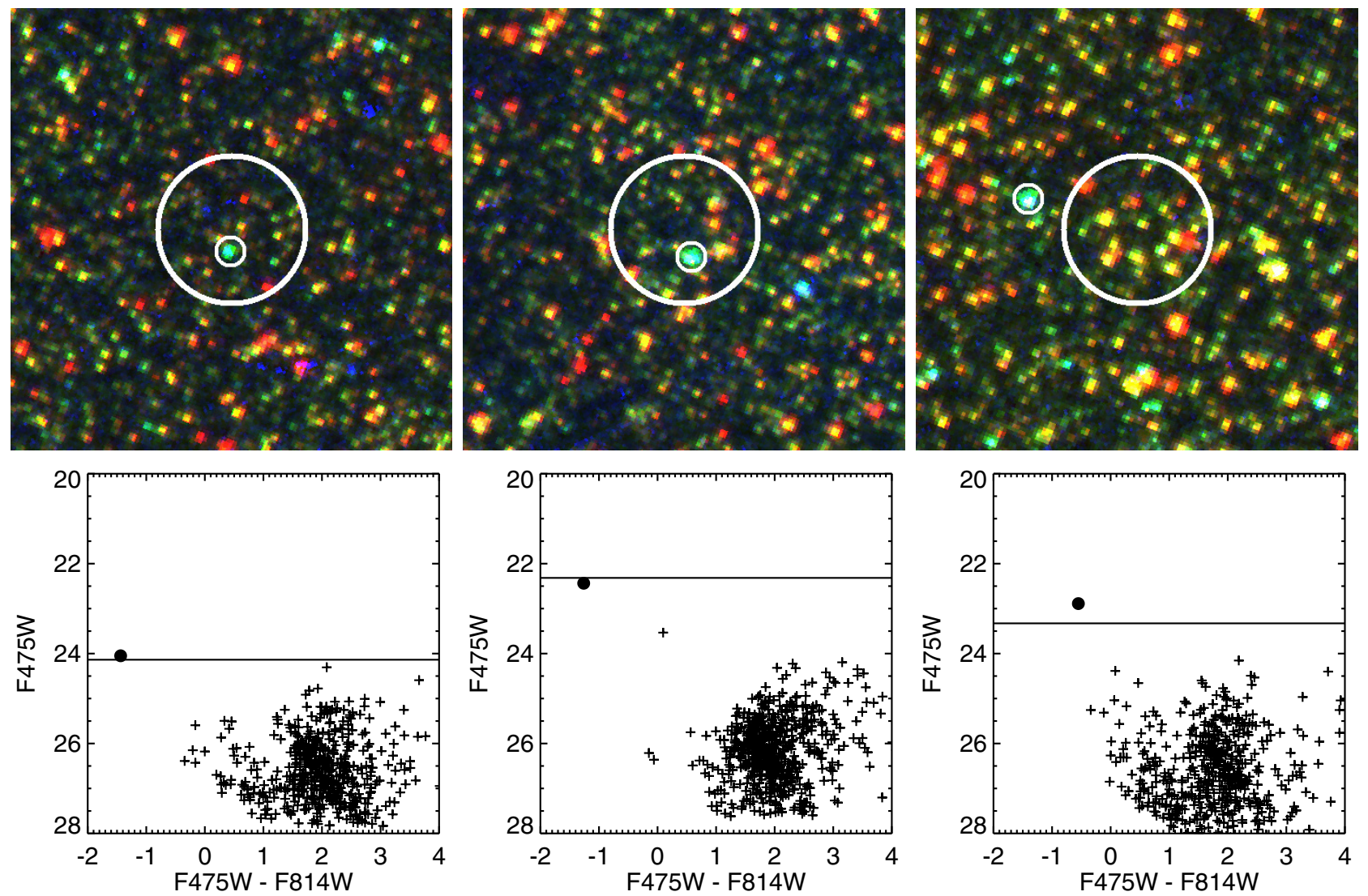

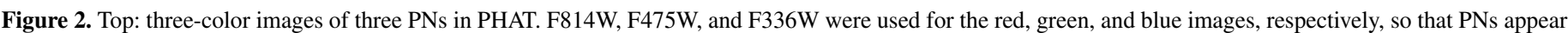

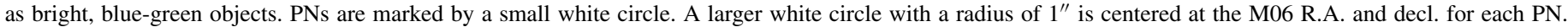

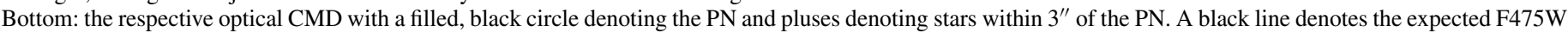

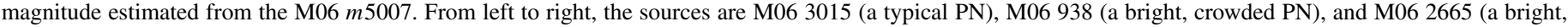
offset PN).

(A color version of this figure is available in the online journal.)

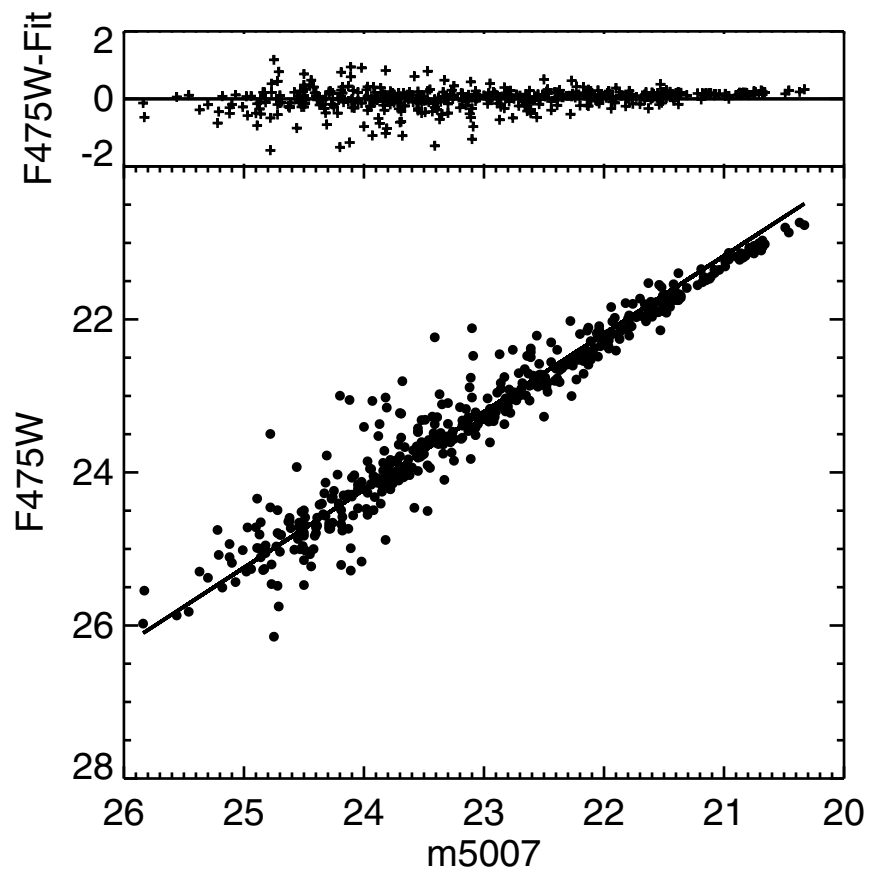

Figure 3. Linear relation between $m 5007$ and F475W magnitudes of the final PHAT PNs catalog. This plot shows the strong dependence of F475W magnitude on $[\mathrm{O}$ III $] \lambda 5007$ line strength. The linear fit was calculated taking into account the quoted M06 $m 5007$ uncertainty as well as the PHAT Poisson errors from F475W photometry.

\subsubsection{Visual Assessment}

To aid in the assessment of our automatic selection method, a visual catalog was created containing a PHAT three-color image, PHAT optical color-magnitude diagram (CMD), LGS [O III] image, and LGS $V$-band image (Massey et al. 2006) for each M06 PN location that PHAT overlaps. The three objects with the lowest $z_{\mathrm{PN}}$ were marked in the PHAT image and CMD. A final PNs catalog was created using the one probable candidate in fields where there was only one probable candidate, and the bluest of the candidates with the three lowest $z_{\text {PN }}$ in the cases where there were multiple probable candidates or no probable candidates. This final catalog of $467 \mathrm{PNs}$ is presented in Section 3.

The visual catalog also allowed for the quick identification of possible misidentifications in the M06 catalog. Locations where there was no probable PN candidate in PHAT and resolved nebulosity in either the PHAT image or in the LGS [O III] image were marked as probable $\mathrm{H}$ II regions. Locations where there was no probable PN candidate in PHAT, no obvious nebulosity, clear $V$-band detection, and a single bright main-sequence (MS) star present in the CMD with F475W much brighter than expected from $m 5007$ were marked as possible stellar emission-line sources, many of which may be WR stars. Both $\mathrm{H}$ II regions and WR stars can have strong [O III] $\lambda 5007$ lines, and could have been detected by M06 as [O III] emission objects. The left two panels of Figure 4 show PHAT images of likely M06 

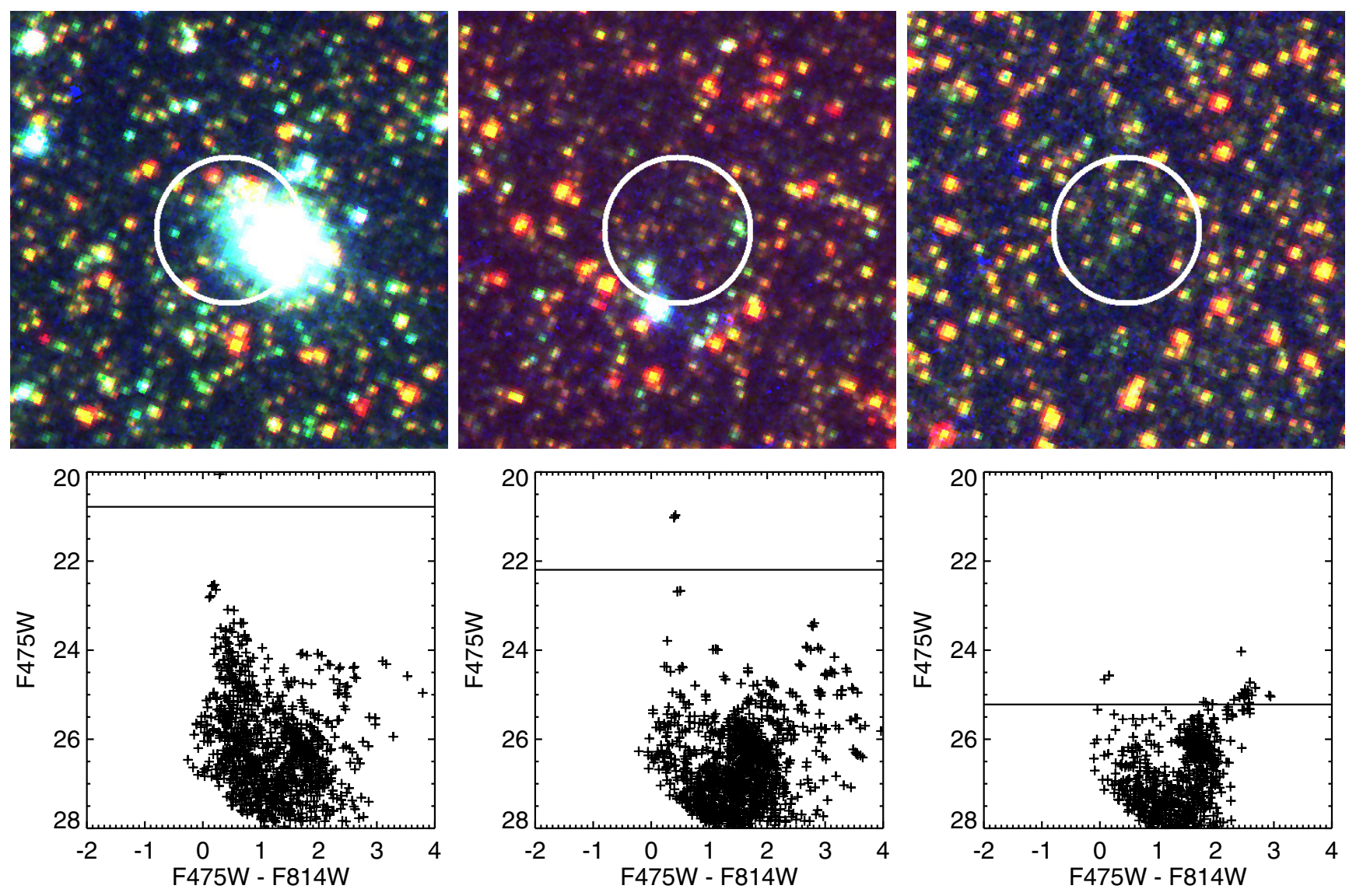

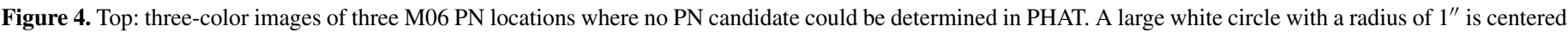

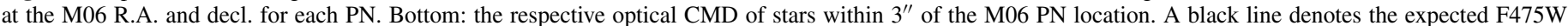

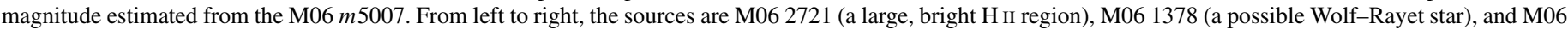
731 (no PN candidate in PHAT).

(A color version of this figure is available in the online journal.)

misidentifications - one marked as an $\mathrm{H}$ II region, the other as a stellar source. A catalog of these possible misidentifications is presented in Section 3. Of the 711 PNs in the M06 catalog that overlap the PHAT images, 152 were likely misidentified.

There were 92 cases where there were no possible PN candidates $\left(z_{\mathrm{PN}}<3\right)$ and no obvious source of misidentification in the PHAT or LGS images. The frequency of these cases is much higher for fainter M06 PNs. For M06 PNs with $m 5007>$ $25 \mathrm{mag}$, more than half were found to have no PN candidate in PHAT and no source of misidentification. The right panel of Figure 4 shows a PHAT image of an M06 PN location with no PN candidate in PHAT. These cases are left out of all catalogs and figures. Figure 5 shows histograms of $m 5007$ magnitude for different PHAT classifications.

\section{RESULTS}

Figure 6 shows the spatial distribution of PNs in the PHAT footprint as well as the locations of possibly misidentified M06 sources. The density of PN sources falls of radially, tracing the old stellar population. Sources tagged as extended $\mathrm{H}$ II regions or other non-PN stellar sources are consistently found near regions of recent and ongoing star formation along the disk characterized by high UV flux. Non-PN objects were selected without prior knowledge of their location.

Table 2 provides astrometric and photometric measurements in six filters ranging from near-IR to near-UV for 467 PNs in the footprint of the current PHAT release. Of the 467 PNs in the catalog, 291 have an F275W measurement, 409 have an F336W measurement, 467 have an F475W measurement, 466 have an F814W measurement, 185 have an F110W measurement, and 156 have an F160W measurement.

Tables 3 and 4 list M06 PNs for which we found a possible source of misidentification. These non-PN objects are listed as either extended $\mathrm{H}$ II regions (Table 3 ) or non-PN stellar sources (Table 4). Astrometry and photometry for stellar sources are included when available. Of these non-PN sources, our classifications for 38 sources agree with spectroscopic classification from Sanders et al. (2012). Most of these are H II regions.

\subsection{Planetary Nebula Luminosity Function}

The planetary nebula luminosity function (PNLF) has been proposed as a standard candle for distance measurements (Ciardullo et al. 1989). The PNLF is described by

$$
N(M 5007) \propto e^{0.307 \times M 5007}\left[1-e^{3\left(M 5007^{*}-M 5007\right)}\right],
$$

where $M 5007^{*}$ is the absolute magnitude bright-end cut off. The same formula can be applied to apparent magnitude when the distances to the PNs are roughly equivalent, as is the case with M31.

The upper half of Figure 7 shows the PNLF of the PHAT PNs catalog using the M06 $m 5007$. The PHAT $m 5007$ PNLF matches the expected form up to $\sim 24$ th magnitude, consistent with the M06 reported completeness of 23.75. The apparent 
Table 2

M31 PNs Photometric Catalog

\begin{tabular}{|c|c|c|c|c|c|c|c|c|c|c|}
\hline M06 ID & PHAT R.A. & PHAT Decl. & $F 275 W$ & $F 336 W$ & $F 475 W$ & $F 814 W$ & $F 110 W$ & $F 160 W$ & $\mathrm{EC}^{\mathrm{a}}$ & $z_{\mathrm{PN}}{ }^{\mathrm{b}}$ \\
\hline 26 & 11.469719 & 42.086807 & $\ldots$ & $\ldots$ & 24.869 & 25.920 & $\ldots$ & $\ldots$ & $>3.8$ & 0.35 \\
\hline 29 & 11.461526 & 42.113659 & $\ldots$ & 26.015 & 24.718 & 25.926 & $\ldots$ & $\ldots$ & $>3.8$ & 0.46 \\
\hline 30 & 11.360975 & 42.142666 & 22.656 & 22.578 & 22.958 & 23.512 & 22.798 & 23.413 & 3.1 & 0.42 \\
\hline 31 & 11.381368 & 42.144012 & 23.235 & 22.673 & 22.097 & 23.865 & 23.051 & 23.487 & $>3.8$ & 0.60 \\
\hline 32 & 11.472247 & 42.147278 & 23.282 & 22.878 & 22.416 & 23.986 & 23.377 & 23.823 & $>3.8$ & 0.52 \\
\hline 33 & 11.372000 & 42.149998 & $\ldots$ & $\ldots$ & 24.827 & 25.762 & 24.550 & 25.225 & $>3.8$ & 0.31 \\
\hline 34 & 11.431614 & 42.156788 & 23.545 & 22.882 & 22.850 & 24.346 & 23.592 & 24.253 & $>3.8$ & 0.57 \\
\hline 44 & 11.530962 & 42.110489 & 22.766 & 22.619 & 22.917 & 23.720 & 23.121 & 23.540 & 3.4 & 0.31 \\
\hline 45 & 11.525844 & 42.116840 & 23.178 & 22.295 & 21.837 & 23.356 & 22.570 & 22.878 & $>3.8$ & 0.49 \\
\hline 46 & 11.508853 & 42.127369 & $\ldots$ & $\ldots$ & 23.712 & 25.093 & $\ldots$ & $\ldots$ & 3.5 & 0.37 \\
\hline : & $\vdots$ & $\vdots$ & $\vdots$ & $\vdots$ & $\vdots$ & $\vdots$ & $\vdots$ & $\vdots$ & $\vdots$ & \\
\hline
\end{tabular}

Notes. Photometric uncertainties of PHAT combined six-band photometry are discussed in Williams et al. (2014).

${ }^{\mathrm{a}}$ Excitation classification $\left(\mathrm{EC} \equiv 0.45 \times\left(F_{5007} / F_{\mathrm{H} \beta}\right)\right.$, for $\left.\mathrm{EC}<5\right)$ as estimated by $\log \left(F_{5007} / F_{\mathrm{H} \beta}\right)=0.7835+0.7003(\mathrm{~F} 475 \mathrm{~W}-m 5007)$.

$\mathrm{b}$ The standard score of the deviation from typical PNs identified in PHAT; see Section 2.2 .2 for full derivation.

(This table is available in its entirety in a machine-readable form in the online journal. A portion is shown here for guidance regarding its form and content.)

Table 3

Misidentified Extended H II Regions

\begin{tabular}{|c|c|c|c|c|c|c|c|}
\hline M06 ID & M06 R.A. & M06 Decl. & M06 m5007 & M06 ID & M06 R.A. & M06 Decl. & M06 $m 5007$ \\
\hline 35 & 11.471667 & 42.159944 & 23.90 & 52 & 11.660417 & 42.182833 & 22.93 \\
\hline 53 & 11.679167 & 42.185806 & 21.31 & 54 & 11.543750 & 42.189306 & 24.33 \\
\hline 55 & 11.638333 & 42.193917 & 20.60 & 56 & 11.535000 & 42.192000 & 21.03 \\
\hline 57 & 11.642917 & 42.195306 & 17.75 & 58 & 11.674167 & 42.196722 & 24.10 \\
\hline 59 & 11.672917 & 42.199167 & 22.39 & 60 & 11.544167 & 42.212194 & 20.97 \\
\hline 62 & 11.680417 & 42.218917 & 24.26 & 64 & 11.645417 & 42.195833 & 22.08 \\
\hline 84 & 11.142083 & 41.954194 & 23.41 & 96 & 11.211250 & 41.936028 & 24.84 \\
\hline 103 & 11.318750 & 41.958861 & 24.43 & 106 & 11.393750 & 41.969889 & 23.70 \\
\hline 109 & 11.366667 & 41.991667 & 24.30 & 117 & 11.291667 & 42.028944 & 22.73 \\
\hline 120 & 11.292917 & 42.041056 & 21.21 & 121 & 11.222500 & 42.042806 & 20.83 \\
\hline 125 & 11.197500 & 41.950194 & 22.46 & 126 & 11.197083 & 41.949361 & 20.92 \\
\hline 139 & 11.435833 & 41.957500 & 21.99 & 147 & 11.637917 & 41.950833 & 23.49 \\
\hline 150 & 11.640417 & 41.986750 & 24.02 & 163 & 11.625833 & 41.987694 & 22.78 \\
\hline 241 & 11.129583 & 41.852500 & 23.86 & 242 & 11.124167 & 41.862889 & 24.08 \\
\hline 243 & 11.158333 & 41.864722 & 22.23 & 246 & 11.125417 & 41.866167 & 22.65 \\
\hline 257 & 11.183750 & 41.899778 & 21.29 & 262 & 11.247917 & 41.920083 & 24.35 \\
\hline 268 & 11.191667 & 41.883028 & 23.50 & 279 & 11.413333 & 41.832139 & 23.39 \\
\hline 285 & 11.405000 & 41.851889 & 21.10 & 295 & 11.434167 & 41.868667 & 21.20 \\
\hline 298 & 11.428750 & 41.876278 & 21.67 & 301 & 11.430417 & 41.883417 & 19.67 \\
\hline 307 & 11.406667 & 41.906722 & 22.84 & 322 & 11.581667 & 41.841889 & 22.11 \\
\hline 324 & 11.573333 & 41.866194 & 19.19 & 327 & 11.555000 & 41.873556 & 21.66 \\
\hline 446 & 11.285000 & 41.660750 & 22.30 & 448 & 11.327083 & 41.670500 & 21.64 \\
\hline 481 & 11.298333 & 41.621306 & 22.08 & 482 & 11.327500 & 41.677833 & 24.12 \\
\hline 494 & 11.431667 & 41.709944 & 22.32 & 659 & 11.252500 & 41.476778 & 20.03 \\
\hline 660 & 11.253333 & 41.478361 & 21.43 & 661 & 11.187083 & 41.478083 & 22.74 \\
\hline 739 & 11.291250 & 41.599917 & 21.51 & 741 & 11.308333 & 41.603917 & 21.42 \\
\hline 954 & 10.913333 & 41.448028 & 20.35 & 996 & 10.979167 & 41.434611 & 21.93 \\
\hline 999 & 11.160000 & 41.419861 & 20.89 & 1000 & 10.982917 & 41.442750 & 21.66 \\
\hline 1020 & 11.197500 & 41.431500 & 21.90 & 1371 & 10.932917 & 41.193583 & 23.21 \\
\hline 1377 & 10.945417 & 41.211056 & 22.46 & 1385 & 11.062917 & 41.258722 & 23.57 \\
\hline 1699 & 10.895000 & 41.164944 & 19.97 & 2159 & 11.675000 & 42.262694 & 24.80 \\
\hline 2581 & 11.564583 & 42.249167 & 24.53 & 2587 & 11.148333 & 41.935139 & 23.22 \\
\hline 2589 & 11.416250 & 41.919278 & 23.11 & 2664 & 11.103750 & 41.627583 & 22.88 \\
\hline 2669 & 11.296250 & 41.612583 & 22.18 & 2673 & 11.301667 & 41.619278 & 20.52 \\
\hline 2674 & 11.299167 & 41.620250 & 20.06 & 2675 & 11.302500 & 41.621250 & 20.82 \\
\hline 2678 & 11.309583 & 41.623639 & 21.73 & 2720 & 11.203333 & 41.453583 & 24.37 \\
\hline 2721 & 11.187500 & 41.464028 & 20.60 & 2722 & 11.181250 & 41.465694 & 24.10 \\
\hline 2994 & 11.244583 & 41.927028 & 20.15 & 3091 & 11.212917 & 41.923278 & 24.28 \\
\hline 3158 & 10.895417 & 41.164583 & 19.86 & 3225 & 11.302083 & 41.621583 & 20.78 \\
\hline
\end{tabular}

Notes. M06 sources that are visually extended in PHAT images and excluded from the PHAT PNs catalog. Most are likely H II regions. (This table is available in its entirety in a machine-readable form in the online journal. A portion is shown here for guidance regarding its form and content.) 


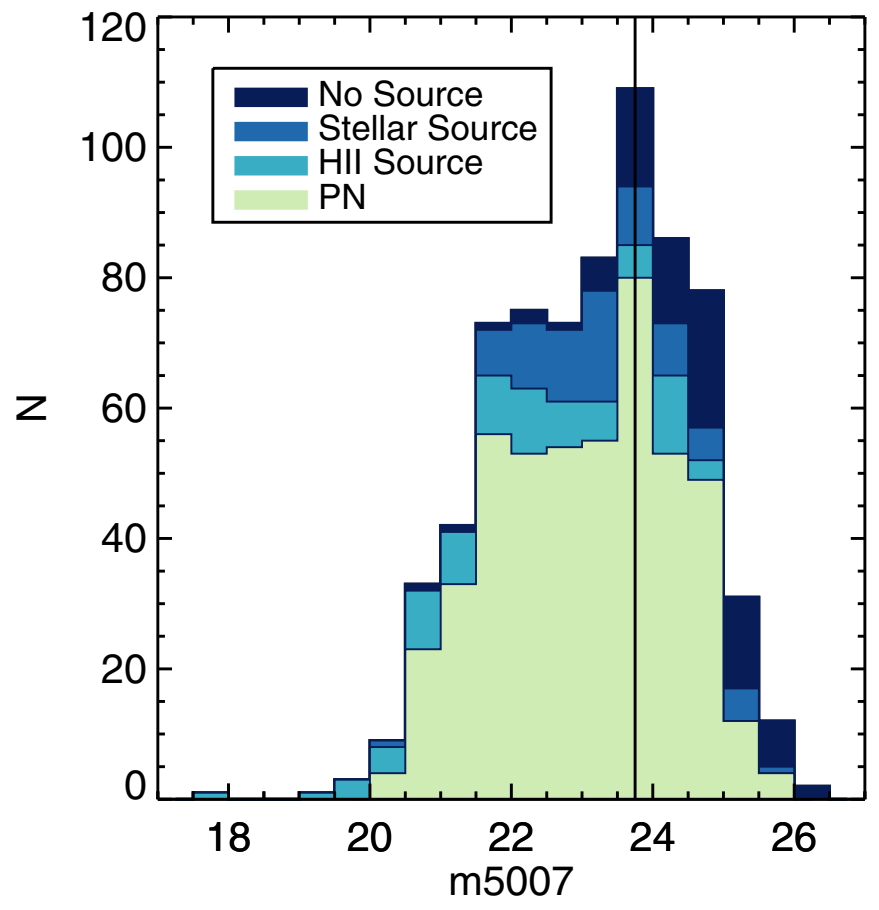

Figure 5. Histograms of visually identified sources for all 711 M06 PNs that fall under the PHAT footprint. The faint end is dominated by fields where no PN could be identified in PHAT and there were no obvious sources of possible misdetection. A black line is drawn at the reported M06 completeness limit of $m 5007=23.75$.

(A color version of this figure is available in the online journal.) magnitude bright-end cut off of the PHAT $m 5007$ PNLF is $m 5007^{*}=20.20 \pm 0.03$, which agrees well with the M06 value of $m 5007^{*}=20.2 \pm 0.1$ and the Ciardullo et al. (1989) value of $m 5007^{*}=20.17$.

The bottom half of Figure 7 shows the PHAT PNLF using F475W magnitudes in place of $m 5007$. As one would expect given Figure 1, the PHAT F475W PNLF is similar in shape to the $m 5007$ PNLF up to $\sim 24$ th magnitude. There is a slight offset in the apparent magnitude bright-end cut off between the $m 5007$ and F475W PNLF and the fainter portion of the F475W PNLF appears flatter. Assuming the F475W PNLF is of the same form as the $m 5007$ PNLF, the apparent magnitude bright-end cut off of the PHAT F475W PNLF is F475W ${ }^{*}=20.69 \pm 0.03$. Assuming a distance modulus of $(m-M)_{0}=24.47 \pm 0.07$ (corresponding to a distance of $785 \pm 25 \mathrm{kpc}$; McConnachie et al. 2005), the absolute magnitude bright-end cut off of the PHAT F475W PNLF is F475W ${ }^{*}=-3.78 \pm 0.08$.

\subsection{CMD Distributions of the PHAT PNs}

We now describe the distribution of PNs in PHAT CMDs; in Section 4.3, we discuss the evolution of PNs through the CMDs.

\subsubsection{Optical}

An optical CMD of PNs compared with nearby stars (Figure 8) confirms previous expectations that most PNs exist in a very blue portion of the CMD where not many stars are expected to be found. In general, PNs are distinctly brighter and bluer than neighboring stars $\left(<3^{\prime \prime}\right)$ as expected from their emission-line-dominated spectrum; however, there is a population of PNs that overlap with MS stars in Figure 8. PNs exist in a distinct region of the optical CMD as outlined by
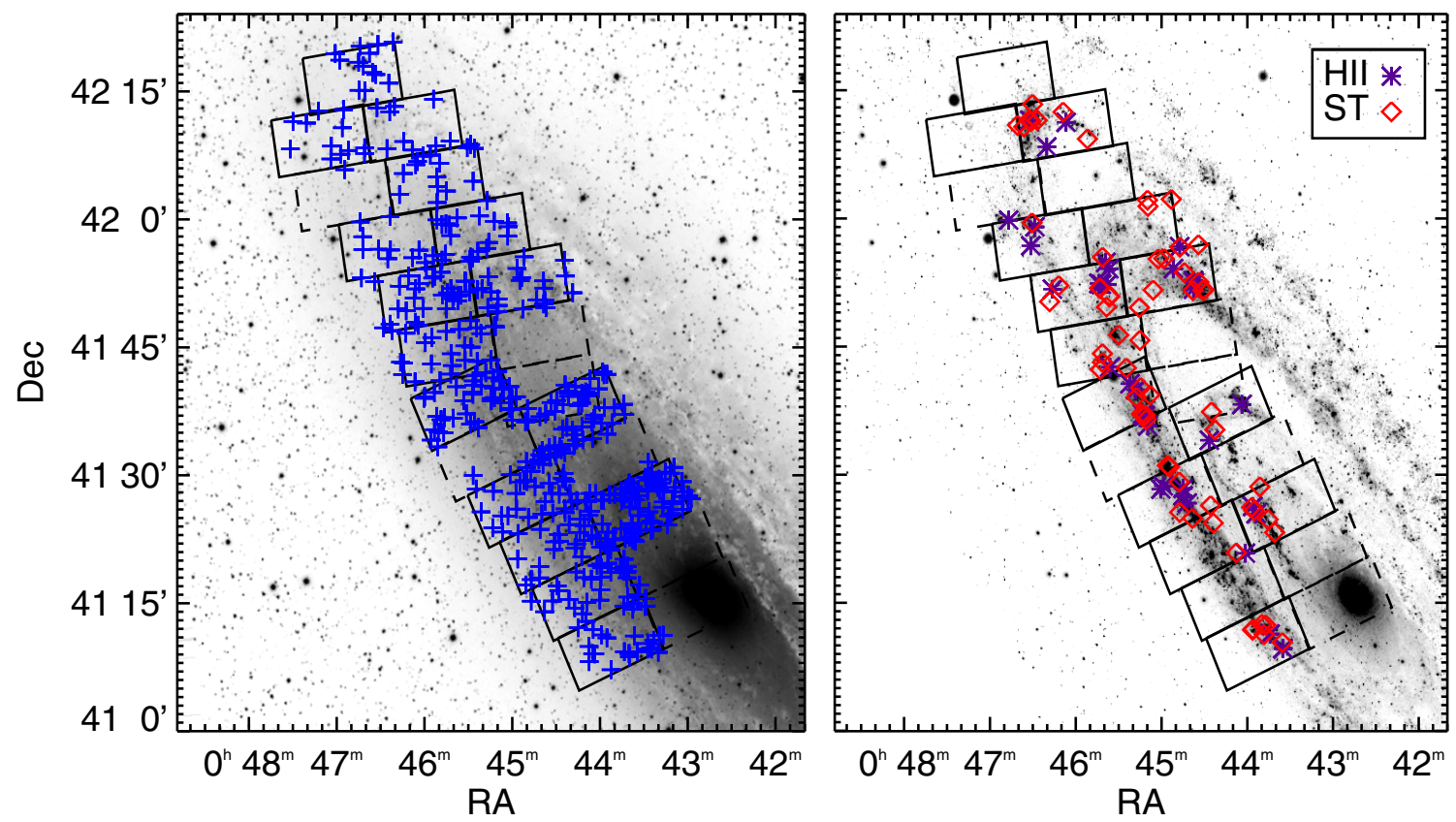

Figure 6. Left: blue crosses denote the location of matched PNs in the PHAT data set over an optical image of M31 (credit and copyright: Martin Pugh http://www.martinpughastrophotography.id.au/). The PHAT bricks currently cross-matched with M06 are outlined in solid black lines. The full PHAT footprint is outlined in dashed black lines. Right: the location of non-PN sources (extended "HII" regions and non-PN "ST"ellar sources) over a Galaxy Evolution Explorer UV image (Thilker et al. 2005). PN spatial density falls off radially while non-PN sources are clustered around UV bright regions. Non-PN objects were rejected without prior knowledge of their location.

(A color version of this figure is available in the online journal.) 
Table 4

Misidentified Stellar Sources

\begin{tabular}{lcccccccc}
\hline \hline M06 ID & PHAT R.A. & PHAT Decl. & $F 275 W$ & $F 336 W$ & $F 475 W$ & $F 814 W$ & $F 110 W$ & $F 160 W$ \\
\hline 65 & 11.592355 & 42.143700 & 19.167 & 19.380 & 20.748 & 20.610 & 20.683 & 20.566 \\
66 & 11.620730 & 42.196083 & 19.415 & 19.446 & 20.632 & 20.228 & 20.124 & 20.020 \\
100 & $\ldots$ & $\ldots$ & $\ldots$ & $\ldots$ & $\ldots$ & $\ldots$ & $\ldots$ & $\ldots$ \\
105 & 11.379783 & 41.964077 & 23.464 & 22.513 & 21.697 & 20.973 & 20.722 & 20.429 \\
111 & 11.204804 & 41.991951 & 22.436 & 22.297 & 23.064 & 22.518 & $\ldots$ & $\ldots$ \\
116 & 11.384217 & 42.012291 & 24.817 & 22.829 & 21.228 & 19.848 & 19.489 & 19.003 \\
127 & 11.369970 & 41.954872 & 20.679 & 20.608 & 21.725 & 21.205 & 21.113 & 20.974 \\
151 & $\ldots$ & $\ldots$ & $\ldots$ & $\ldots$ & $\ldots$ & $\ldots$ & $\ldots$ & $\ldots$ \\
152 & 11.703743 & 42.000370 & 21.215 & 21.010 & 21.024 & 19.468 & $\ldots$ & $\ldots$ \\
153 & 11.630049 & 42.009483 & 22.363 & 21.906 & 22.805 & 22.122 & $\ldots$ & $\ldots$ \\
$\vdots$ & $\vdots$ & $\vdots$ & $\vdots$ & $\vdots$ & $\vdots$ & $\vdots$ & $\vdots$ & $\vdots$ \\
\hline
\end{tabular}

Notes. M06 sources that are likely misidentified non-PN stellar sources and excluded from the PHAT PNs catalog. PHAT R.A., decl., and photometry is included when available. Photometric uncertainties of PHAT combined six-band photometry are discussed in Williams et al. (2014).

(This table is available in its entirety in a machine-readable form in the online journal. A portion is shown here for guidance regarding its form and content.)
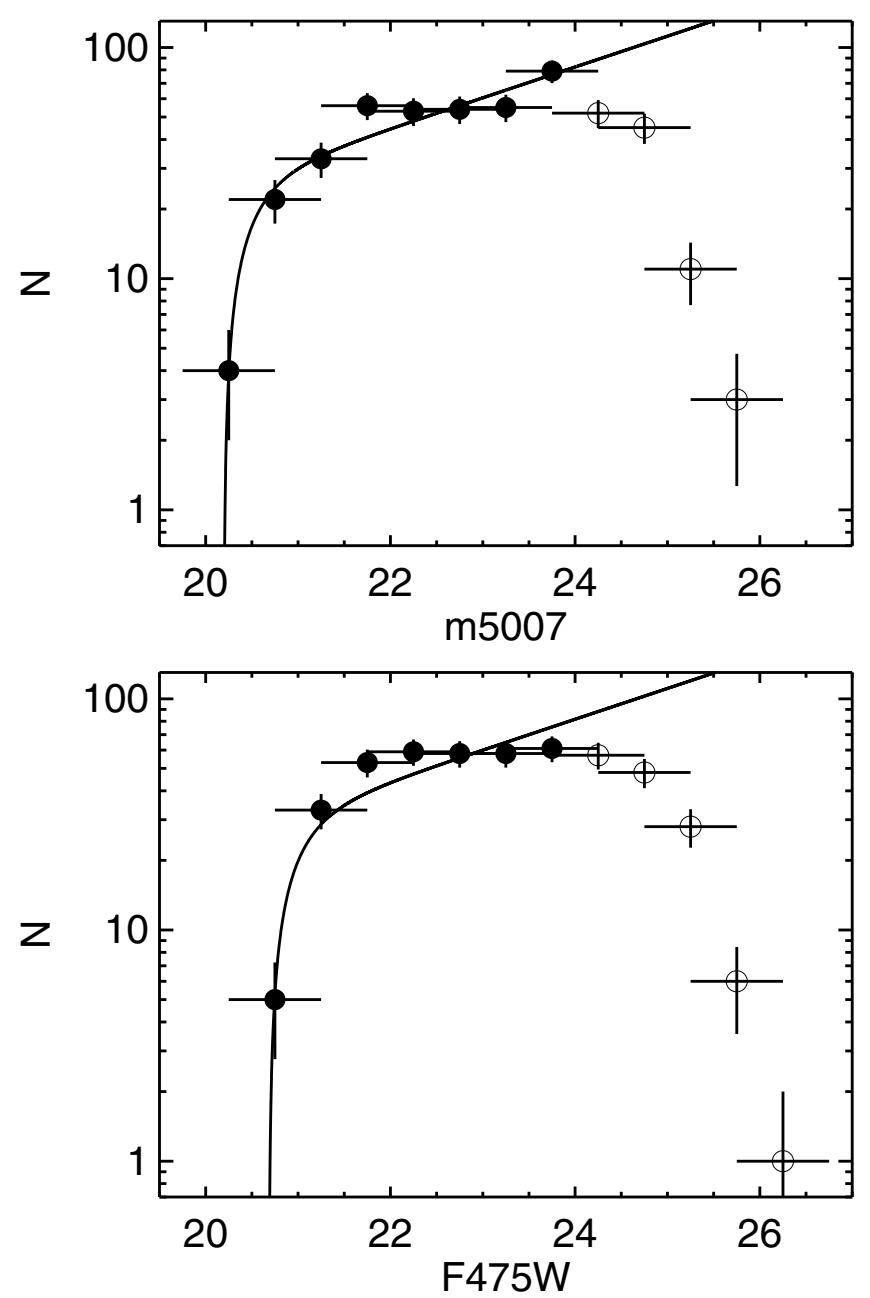

Figure 7. PN luminosity function of the PHAT PNs catalog using $m 5007$ (top) and F475W (bottom). The solid line shows the best fit to the PNLF as described by Equation (3). Filled circles denote values brighter than the completeness limit of 24th magnitude. Open circles denote values fainter than 24th magnitude, which were not used in the fit.

the parallelogram in Figure 8, with hand-drawn boundaries $-1.8<\mathrm{F} 475 \mathrm{~W}-\mathrm{F} 814 \mathrm{~W}<0.2$ and $22>\mathrm{F} 814 \mathrm{~W}<27$, containing $95 \%$ of our PNs sample.

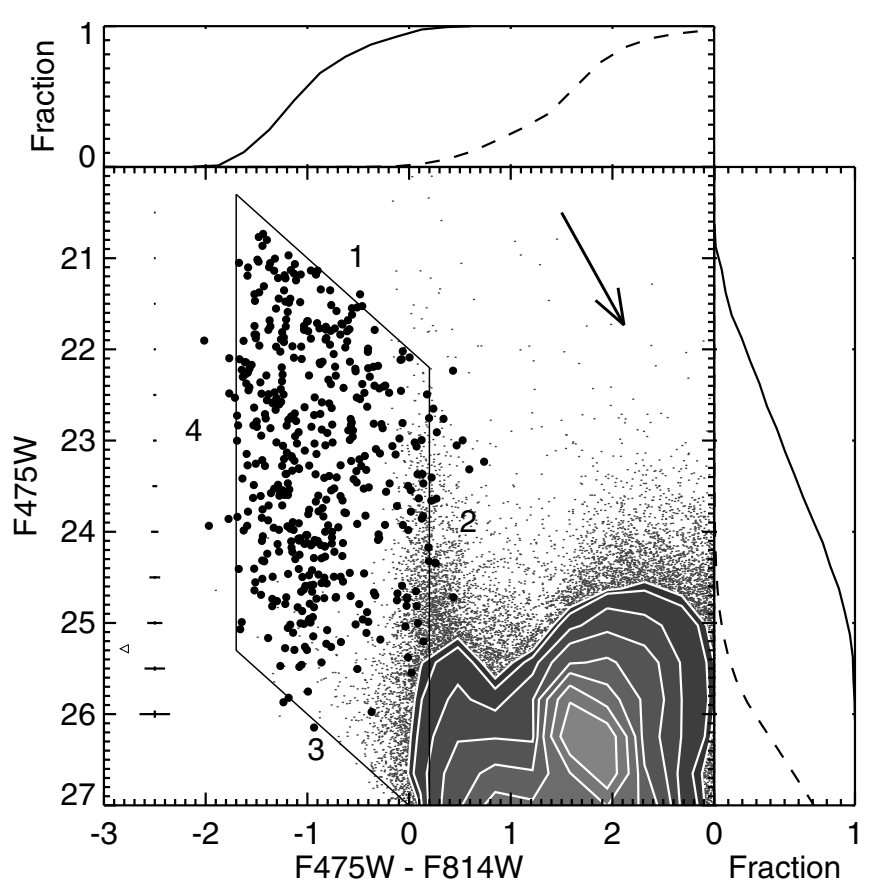

Figure 8. Black circles denote PNs. Background gray dots and contours indicate stars within $3^{\prime \prime}$ of a PN. An open triangle at $(-2.8,25.3)$ represents the F475W magnitude of the one PN with $\mathrm{F} 814 \mathrm{~W} \mathrm{~S} / \mathrm{N}<4$. A rhomboid with sides labeled $1,2,3$, and 4 outlines the boundaries of the PNs population. Crosses along the left side of the plot indicate median Poisson errors from the photometry of PNs binned by F475W. A reddening vector shows the extinction effect of $A_{V}=1$. Above: the normalized cumulative distribution of F475W-F814W for PNs (solid line) and stars within $3^{\prime \prime}$ of a PN (dashed line). Right: the normalized cumulative distribution of F475W for PNs (solid line) and stars within $3^{\prime \prime}$ of a PN (dashed line). PNs exist in a unique bright blue region of PHAT optical CMDs.

\subsection{2. $U V$}

More than $87 \%$ of PNs in the PHAT catalog have detections in F336W and $62 \%$ have detections in both UV filters. The left side of Figure 9 shows the locations on the optical CMD of PNs with and without UV detections. UV detection is much more common among PNs that are bright in F475W, as would be expected for flux due to stellar continuum with some contribution from 


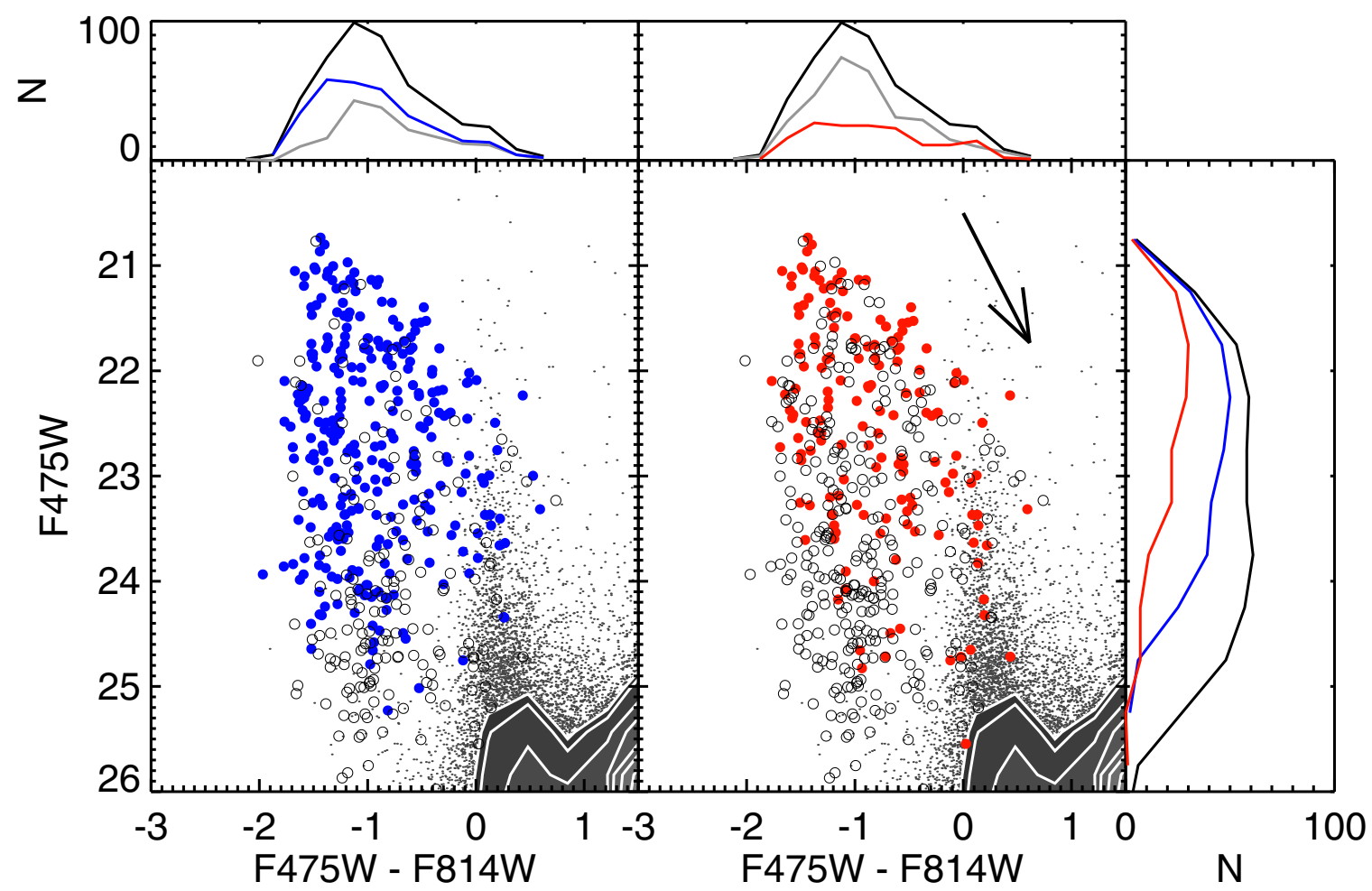

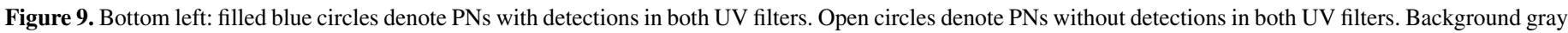

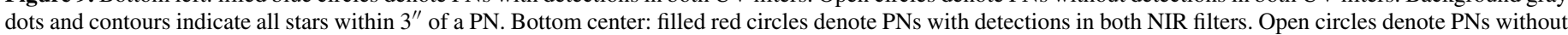

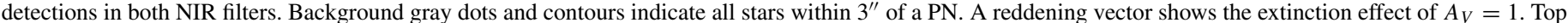

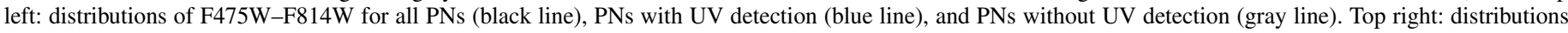

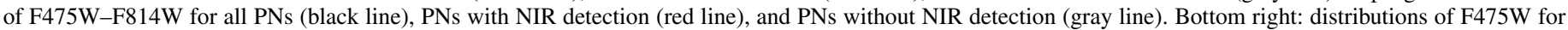
all PNs (black line), PNs with UV detection (blue line), and PNs with NIR detection (red line). UV and NIR detection is more common for PNs bright in F475W.

(A color version of this figure is available in the online journal.)

emission lines. UV detection is also slightly more common among bluer PNs, as the brightest PNs also tend to be the bluest.

Figure 10 shows the UV CMD of the PNs with detections in both UV filters. PNs tend to be brighter and redder in the UV than other stars within $3^{\prime \prime}$. However, these neighboring stars are dominated by MS stars and there is only a slight color difference between PNs and the population of hot MS stars, as would be expected from the assumption that UV flux of PNs comes from the very hot central star. The brightest PNs have wellconstrained colors, while the spread in color increases for fainter F275W magnitudes that tend to be the noisiest measurements in the PHAT filter set.

\subsubsection{NIR}

More than $33 \%$ of PNs in our catalog have detections in both NIR filters. Nearly all PNs with detections in the NIR also have detection in F336W, 84\% of which have detections in both UV filters. Thus, any PN bright enough to be detected in the NIR is almost certainly also detected in the UV, where proportionally more of the PN's bolometric flux is emitted. The right side of Figure 9 shows the locations on the optical CMD of PNs with and without NIR detections. Similar to the UV, NIR detection is much more common among PNs that are bright in F475W. However, the optical color distribution of PNs with detections in NIR is relatively flat and distinctly different from the optical color distribution of all PNs.

Figure 11 shows the NIR CMD of the PNs with detections in both NIR filters. PNs are distinctly bluer and brighter in the
NIR than stars around them, which are primarily cool red giant branch (RGB) and AGB stars. Their distinct color in the NIR is due to the few emission lines in F110W and the lack of emission lines in F160W.

There are two NIR-bright PNs in the PHAT catalog with F110W - F160W $>1$ that are separate from the rest of the PNs population. It is likely that the PN in each case is coincident with an NIR-bright source.

PNs are indeed unique in their photometric signal across all of PHAT's six bands-particularly their optical and NIR color. However, their most distinguishing feature by far is a booming F475W signal best probed by F475W-F814W color. Like in F475W, emission lines in F110W add additional flux that give PNs distinct NIR colors. However, as seen in Figure 12, PNs are best secluded by their optical color. Any diagnostic that used multiple bands to identify PNe would necessarily have most of its weight on $\mathrm{F} 475 \mathrm{~W}$, as this is where the ratio of line emission to stellar continuum is largest (see Section 4.4).

\subsection{Excitation Classification}

Since PNs are emission-line objects, we assume the majority of F475W flux is from emission lines, allowing us to estimate line flux ratios from the difference between the $\mathrm{F} 475 \mathrm{~W}$ and $m 5007$ magnitudes. As seen in Figure 1, the three strongest emission lines that fall under $\mathrm{F} 475 \mathrm{~W}$ are [O III] $\lambda 5007$, [O III] $\lambda 4959$, and $\mathrm{H} \beta$. Because the [O III] $\lambda 5007$ to [O III] $\lambda 4959$ ratio is nearly constant for all PNs, differences in the $m 5007-\mathrm{F} 475 \mathrm{~W}$ color of our PNs are largely due to differences in the [O III] to $\mathrm{H} \beta$ 


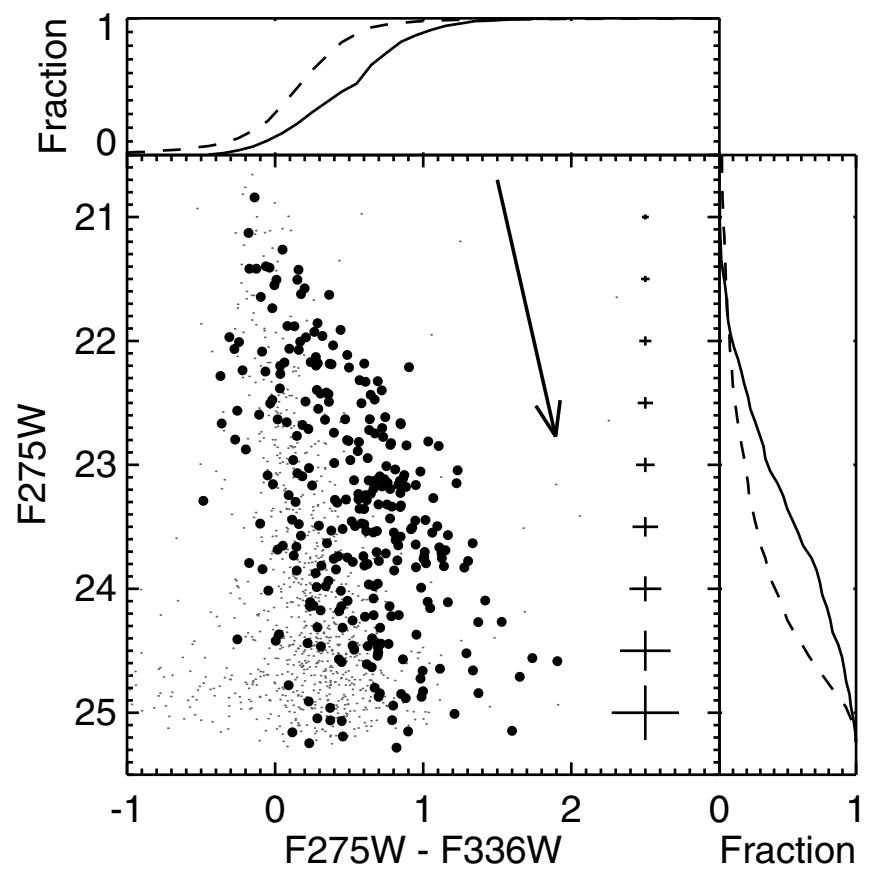

Figure 10. Filled black circles denote PNs. Background gray dots indicate stars within $3^{\prime \prime}$ of a PN. Crosses along the right side of the plot indicate median Poisson errors from the photometry of PNs binned by F275W. A reddening vector shows the extinction effect of $A_{V}=1$. Top: the normalized cumulative distribution of F275W-F336W for PNs (solid line) and stars within $3^{\prime \prime}$ of a PN (dashed line). Right: the normalized cumulative distribution of F275W for PNs (solid line) and stars within $3^{\prime \prime}$ of a PN (dashed line). There are only slight differences between PNs and the population of hot MS stars in the PHAT UV CMD.

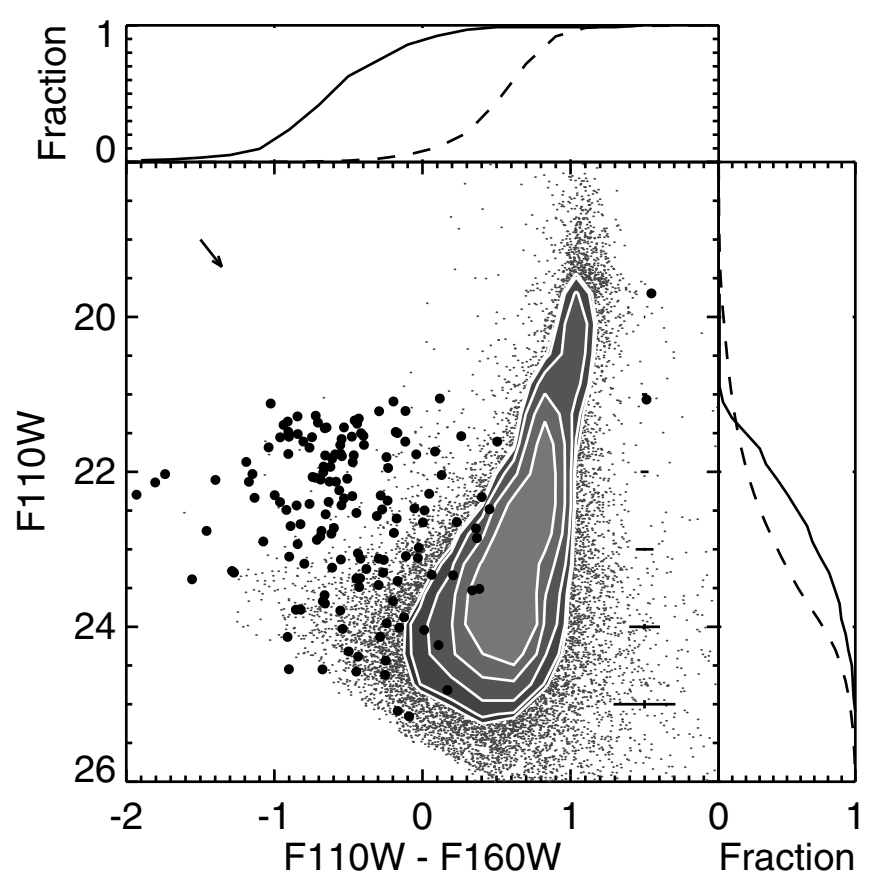

Figure 11. Filled black circles denote PNs. Background gray dots and contours indicate stars within $3^{\prime \prime}$ of a PN. Crosses along the right side of the plot indicate median Poisson errors from the photometry of PNs binned by F110W. A reddening vector shows the extinction effect of $A_{V}=1$. Top: the normalized cumulative distribution of F110W-F160W for PNs (solid line) and stars within $3^{\prime \prime}$ of a PN (dashed line). Right: the normalized cumulative distribution of F110W for PNs (solid line) and stars within $3^{\prime \prime}$ of a PN (dashed line). PNs are bluer and brighter in the NIR than neighboring cool RGB and AGB stars.

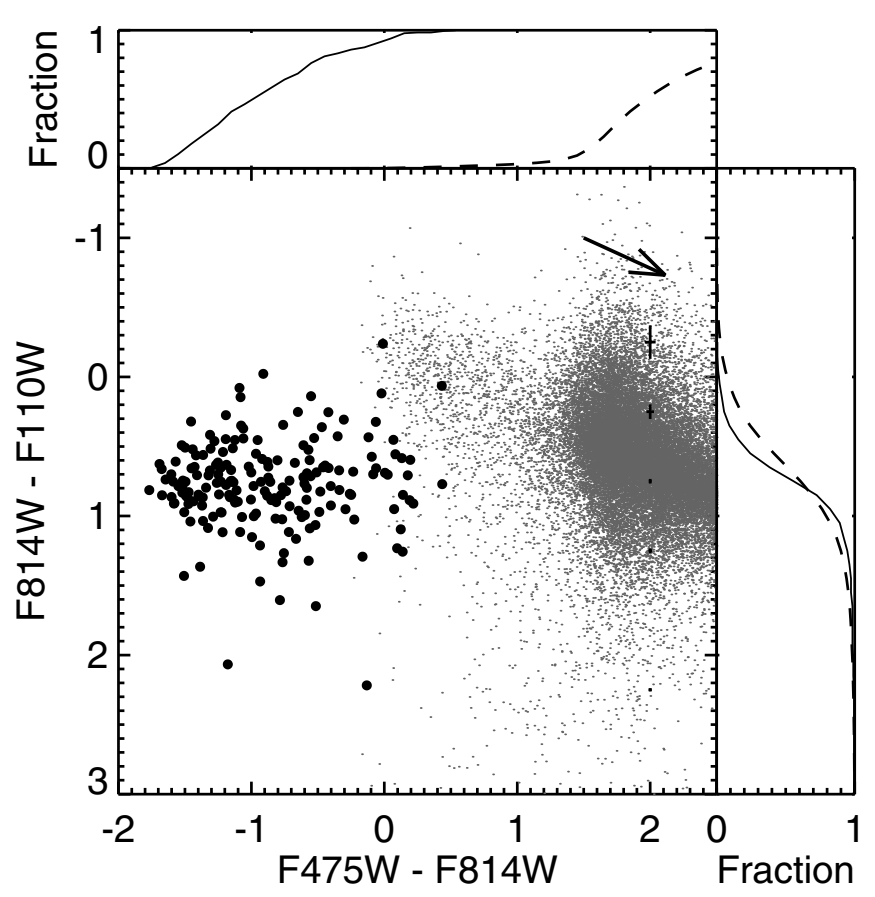

Figure 12. Filled black circles denote PNs. Background gray dots indicate stars within $3^{\prime \prime}$ of a PN. Crosses along the right side of the plot indicate median Poisson errors from the photometry of PNs binned by F814W-F110W. A reddening vector shows the extinction effect of $A_{V}=1$. Top: the normalized cumulative distribution of F475W-F814W for PNs (solid line) and stars within $3^{\prime \prime}$ of a PN (dashed line). Right: the normalized cumulative distribution of F814W-F110W for PNs (solid line) and stars within $3^{\prime \prime}$ of a PN (dashed line). PNs are best distinguished from stars by their optical color.

ratio. This ratio is a measure of ionization in the nebula and, in general, is expected to be large when the central star is very hot.

To explore the possibility of using F475W $-m 5007$ as a probe of the $[\mathrm{O} \mathrm{III}] / \mathrm{H} \beta$ emission-line ratio, we used data from Sanders et al. (2012), who have published spectral line ratio measurements for several PNs in M31, 69 of which are in the PHAT catalog and have both $\mathrm{H} \beta$ and [O III] $\lambda 5007$ measurements. Figure 13 shows the relation between $\mathrm{F} 475 \mathrm{~W}-$ $m 5007$ and the $[\mathrm{O} \mathrm{III}]$ to $\mathrm{H} \beta$ ratio, $F_{5007} / F_{\mathrm{H} \beta}$. The large spread of the relation is likely due to differences in the underlying stellar continuum, which can significantly contribute to the total F475W flux when line emission is comparatively weak. However, a relation between the relative F475W $-m 5007$ and $F_{5007} / F_{\mathrm{H} \beta}$ exists. For low values of $F_{5007} / F_{\mathrm{H} \beta}(<11)$, i.e., comparatively strong $\mathrm{H} \beta$ lines, the relation is quite strong, such that PNs with lower $F_{5007} / F_{\mathrm{H} \beta}$ values have brighter $\mathrm{F} 475 \mathrm{~W}$ magnitudes in relation to their $m 5007$ magnitudes. At higher values of $F_{5007} / F_{\mathrm{H} \beta}$, i.e., comparatively weak $\mathrm{H} \beta$ lines, the relation saturates as the contribution of $\mathrm{H} \beta$ to the total flux becomes indistinguishable from the continuum and weaker emission lines. Therefore, estimates of $F_{5007} / F_{\mathrm{H} \beta}$ can only be made for PNs with F475W $-m 5007<0.2$. Estimates of the $F_{5007} / F_{\mathrm{H} \beta}$ ratio can be made for 170 of the 467 PNs in the PHAT catalog using Equation (4):

$$
F_{5007} / F_{\mathrm{H} \beta}=10^{0.7835+0.7003(\mathrm{~F} 475 \mathrm{~W}-m 5007)} .
$$

The ratio $F_{5007} / F_{\mathrm{H} \beta}$ has been used to calculate the excitation classification (EC) of low EC PNs $(\mathrm{EC}<5$; Dopita \& Meatheringham 1990; Reid \& Parker 2010). We adopt the $\operatorname{Ex}_{\rho}$ method from Reid \& Parker (2010) for calculating the EC of 


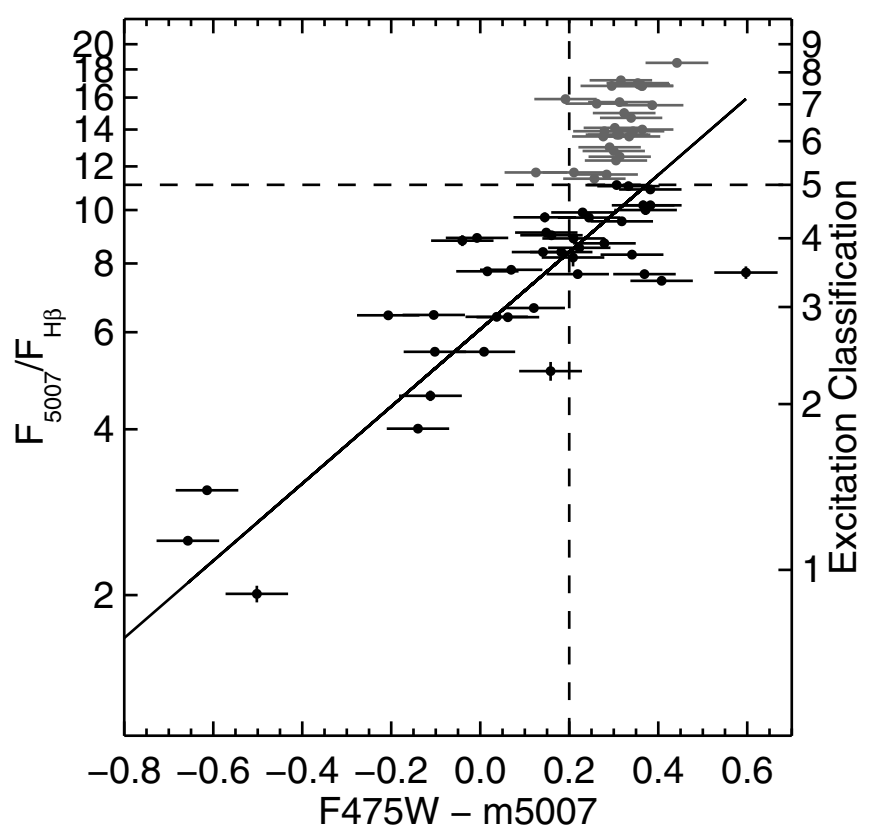

Figure 13. Relationship between the difference of F475W and $m 5007$ magnitudes and the $F_{5007} / F_{\mathrm{H} \beta}$ line ratio and excitation classification (EC $\equiv$ $0.45\left(F_{5007} / F_{\mathrm{H} \beta}\right)$ for low-EC PNs) using emission-line flux ratios from Sanders et al. (2012). Black points denote low EC PNs $(\mathrm{EC}<5)$, for which $\mathrm{H} \beta$ makes a significant contributions to $\mathrm{F} 475 \mathrm{~W}$ flux. Gray points denote mediumto high-EC PNs $(\mathrm{EC}>5$ ), for which the EC cannot be accurately calculated from $F_{5007} / F_{\mathrm{H} \beta}$. A solid line denotes a linear least-squares fit between $\mathrm{F} 475 \mathrm{~W}-m 5007$ and $\log \left(F_{5007} / F_{\mathrm{H} \beta}\right),(\mathrm{RMSE}= \pm 2.0)$. The data has a correlation coefficient of 0.9 . The largest source of error is the uncertainty in $m 5007$ of $0.07 \mathrm{mag}$. Errors in $F_{5007} / F_{\mathrm{H} \beta}$ are often smaller than the plot symbols. Dashed lines indicate where $\mathrm{F} 475 \mathrm{~W}-m 5007=0.2$ and where $\mathrm{EC}=5$. The majority of PNs with $\mathrm{F} 475 \mathrm{~W}-m 5007<0.2$ also have $\mathrm{EC}<5$.

low EC PNE as defined in Equation (5):

$$
\mathrm{EC} \equiv 0.45 \times\left(F_{5007} / F_{\mathrm{H} \beta}\right)
$$

EC estimates for the 170 PNs with F475W $-m 5007<0.2$ are included in Table 2 with an uncertainty of $\sigma_{\mathrm{EC}}= \pm 1$. All PNs with $\mathrm{F} 475 \mathrm{~W}-m 5007 \geqslant 0.2$ are assumed to have EC $>3.8$. As excitation increases, the contributions of other emission lines such as $\mathrm{He}$ II become comparable with $\mathrm{H} \beta$, which would cause an underestimate in the PN's EC.

\section{DISCUSSION}

\subsection{Extinction}

M31 is known to be a dusty galaxy (e.g., Draine et al. 2014; Dalcanton et al. 2014). Therefore, we need to understand how dust may be affecting our PNs photometry. To determine the degree to which the PNs sample is attenuated by extinction, we selected a subsample of PNs from "dust-free" regions as determined from the extinction maps of Dalcanton et al. (2014). These PNs were selected to be in regions with $A_{V}<0.5$ and a low fraction of reddened stars. Their optical CMD is shown in Figure 14, and the distribution is qualitatively equivalent to that of the full sample. Perhaps because PNs tend not to be associated with ongoing star formation or because PNs are associated with old stars $(>1 \mathrm{Gyr})$ that have migrated out of the galactic plane, our PNs photometry appears to be relatively unaffected by dust extinction.

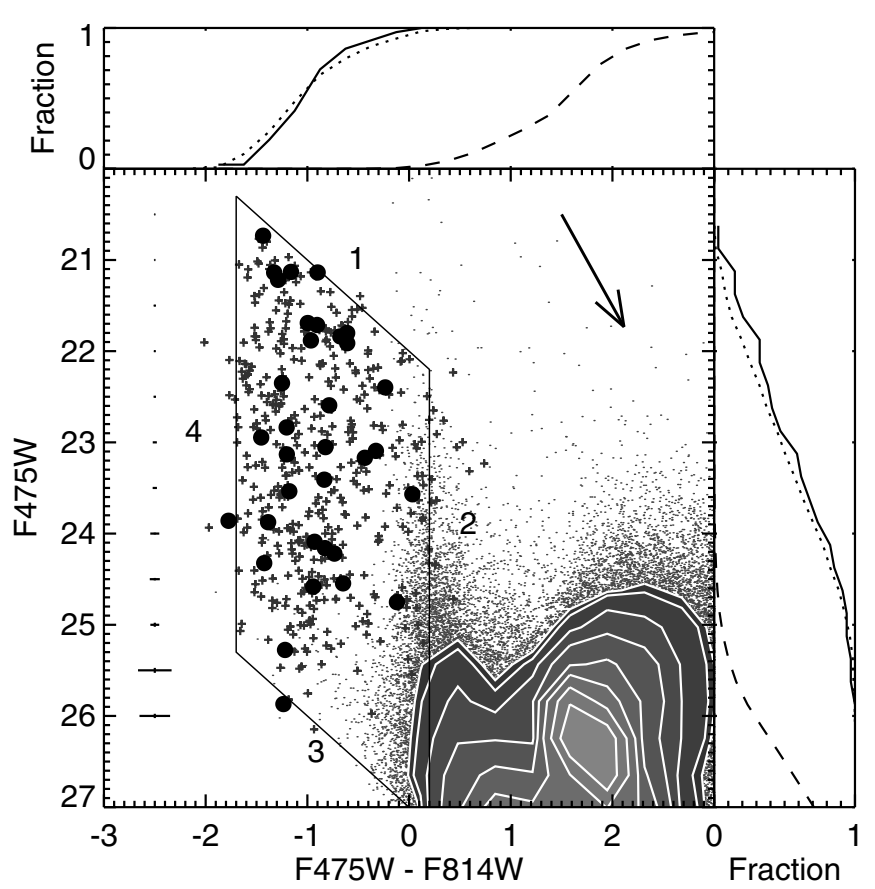

Figure 14. Same as Figure 8, but now large black circles and solid lines denote only PNs from "dust-free" regions. Gray crosses and dotted lines denote the full sample. The distribution is qualitatively very similar to that of the full sample, suggesting the PHAT PNs catalog does not suffer large differential extinction.

\subsection{PNLF}

The consistency of the PHAT $m 5007$ PNLF with the M06 PNLF suggests that the PHAT PNs catalog is a representative and unbiased subset of the M06 catalog. Despite the fact that the F475W bandpass covers emission lines other than [O III] $\lambda 5007$ and continuum from the central star, the PHAT F475W PNLF is similar in form to the $m 5007$ PNLF, particularly on the bright end, aside from a slight offset. This is because the contribution by stellar continuum and weaker emission lines to the total F475W flux is less significant in brighter PNs, for which the F475W magnitude is a good indicator of $m 5007$, making the F475W PNLF a possible proxy for the traditional $m 5007$ PNLF for future extragalactic surveys. The flatness of the faint end of the PHAT F475W PNLF could be due to the larger relative contribution by stellar continuum for PNs with weaker [O III] emission.

\subsection{Excitation Classification and PN Evolution}

Excitation classification has been found to depend on the evolutionary state of a PN, which is reflected in the central star's effective temperature, the PN's radius, and the PN's expansion velocity (Dopita et al. 1987, 1988; Dopita \& Meatheringham 1990, 1991a, 1991b; Gurzadyan \& Egikian 1991; Reid \& Parker 2010). Because many parameters affect the EC, the correlations between EC and physical properties of PNs have large scatter that, coupled with the large uncertainties of our own EC estimates, means we cannot reliably estimate individual parameters. However, our ability to isolate a subsample of low EC PNs allows us to place some PNs in the evolutionarily young phase with small sizes and low central star temperatures. For the remainder of this paper, we referred to PNs with EC below the upper limit of our ability to determine EC $(\mathrm{EC}<3.8)$ as low-EC PNs and all other PNs as medium- to high-EC PNs; note that the canonical definition of "low EC" includes up to 
an EC of five. We do expect some cross-contamination due to the large uncertainties in our F475W $-m 5007$ measurements such that some PNs in our sample of low-EC PNs are in fact medium- to high-EC PNs and vice versa. In addition, some high-EC PNs may be misclassified as low-EC PNs due to increased contribution of $\mathrm{He}$ II and other weak lines in the F475W bandpass.

According to PN evolutionary tracks based on photoionization models described in Dopita \& Meatheringham (1990) and Reid \& Parker (2010), all PNs, regardless of their initial mass, begin as low-EC PNs. They then increase in EC as they evolve to a mass-dependent maximum EC before returning to a slightly lower EC. Only lower-mass PNs (central star stellar mass $\lesssim 0.6 M_{\odot}$ ) are expected to return to an EC lower than five. The majority of low-EC PNs in our catalog are therefore likely to be young, but with a wide range of initial masses. Additionally, high-EC PNs in our catalog are expected to cover the entire range of PNs ages except for the youngest.

Another set of PN evolutionary models useful for understanding how PNs evolve through PHAT color-magnitude and color-color diagrams are one-dimensional radiationhydrodynamic models that describe the evolution of PNs by modeling a circumstellar envelope along with the central star as it evolves from the AGB toward the white-dwarf cooling path (Perinotto et al. 2004). Studies of the emission properties of these models (e.g., Schönberner et al. 2007; Méndez et al. 2008) explain that in early stages of evolution, PNs increase in $m 5007$ brightness as they increase in EC until they reach a maximum $m 5007$ brightness. The intensity and duration of maximum m5007 brightness is mass dependent. In general, higher-mass progenitors result in brighter maximum $m 5007$ but for shorter duration than fainter low-mass progenitors (Kwok 2000). Maximum $m 5007$ brightness is reached at ECs higher than the upper limit of our ability to determine EC of PNs in PHAT. Therefore, we expect the PNs in our catalog to evolve from low EC to high EC simultaneously increasing in $m 5007$ brightness to a maximum brightness. Indeed, this is the qualitative behavior observed in Figure 15, as described below.

\subsubsection{PN Evolution through CMDs}

Figure 15 shows the locations of low-EC and medium- to high-EC PNs on an optical CMD. We see a bifurcation of lowEC and medium- to high-EC PNs among PNs with F475W $<23.5$. This behavior would be consistent with the evolutionary transition of a high-mass progenitor along or parallel to side 1 (the upper boundary in the figure) as the PN's [O III] $\lambda 5007$ flux reaches a maximum. The proposed transition aligns well with the PN evolutionary models described above, namely a transition from low EC to higher EC and from fainter F475W magnitudes to the brightest. Brightening in F475W along side 1 toward the bluer portion of the CMD corresponds to evolution with a constant F814W magnitude.

The less distinct red boundary indicated by side 2 in Figure 15 is made up of mostly low EC PNs and overlaps MS stars. The overlap with the MS is most likely due to interstellar reddening of young PNs but could also indicate residual contamination from WR stars. The faint boundary (side 3) and the similar boundary for field stars are products of the cuts in signal-tonoise.

Figure 16 shows the locations of low-EC and medium- to high-EC PNs on a UV CMD. The low-EC PNs are systematically brighter and bluer, suggesting some transition toward redder and fainter UV magnitudes as they evolve into higher

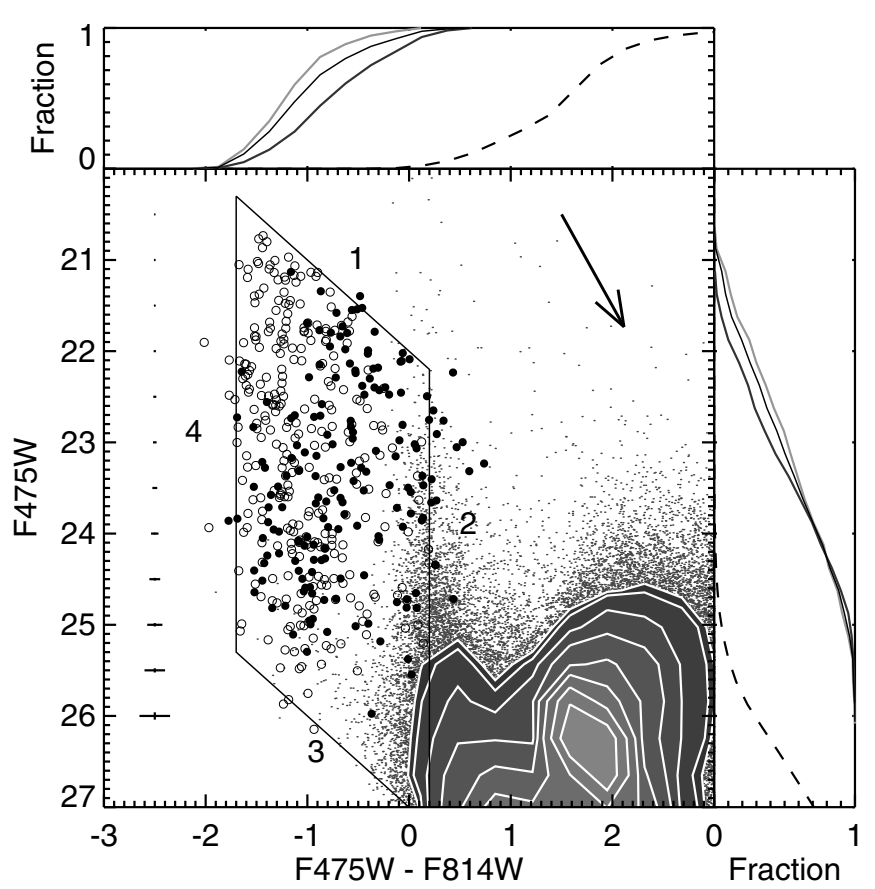

Figure 15. Filled black circles denote low-EC PNs. Open circles denote medium- to high-EC PNs. Background gray dots and contours indicate stars within $3^{\prime \prime}$ of a PN. A rhomboid with sides labeled 1, 2, 3, and 4 outlines the boundaries of the PNs population. Crosses along the left side of the plot indicate median Poisson errors from the photometry of PNs binned by F475W. A reddening vector shows the extinction effect of $A_{V}=1$. Top: the normalized cumulative distribution of F475W-F814W for PNs (thin black line), low-EC PNs (dark gray line), medium- to high-EC PNs (light gray line), and stars within $3^{\prime \prime}$ of a PN (dashed line). Right: the normalized cumulative distribution of F475W for PNs (thin black line), low-EC PNs (dark gray line), medium- to high-EC PNs (light gray line), and stars within $3^{\prime \prime}$ of a PN (dashed line). There is a distinct bright blue population of medium- to high-EC PNs, suggesting an evolutionary transition to higher F475W flux as increasing central star temperature increases excitation in the nebula.

ECs. The slightly redder extant of the PN distribution compared to bright MS stars is likely due to increased contribution from nebular continuum in the F336W bandpass. Also shown in Figure 16 are two post-AGB (P-AGB) evolutionary tracks from Vassiliadis \& Wood (1993) converted to WFC3/UVIS photometry as described in Girardi et al. (2008), shown without extinction correction. We see an increased spread of UV color at fainter F275W magnitudes, but it is hard to say for certain if the spread represents the true spread in PNs UV color or is due to photometric uncertainty and/or interstellar reddening. Still, it is clear that reduced UV flux is a prominent difference between low-EC and medium- to high-EC PNs on the UV CMD. Such a transition is expected from P-AGB evolutionary tracks.

Figure 17 shows the CMD for F336W-F475W versus F336W. Because F336W is dominated by stellar and nebular continuum and F475W by line emission, this diagram compares emissionline flux to underlying continuum. Low-EC PNs appear much like hot stars, but medium- to high-EC PNs show up where few stars are expected to be found. The increased excitation increases flux in the emission-line-dominated F475W bandpass, pulling PNs redward to a relatively unpopulated portion of the CMD. This diagnostic may be useful for future studies of PNs as it is possible to replicate through ground-based observations. Of the six PHAT filters, PNs are brightest in F336W and F475W.

In the NIR, there is no separation in either color or magnitude between low-EC and medium- to high-EC PNs. MS stars in PHAT are expected to have NIR colors redder than 


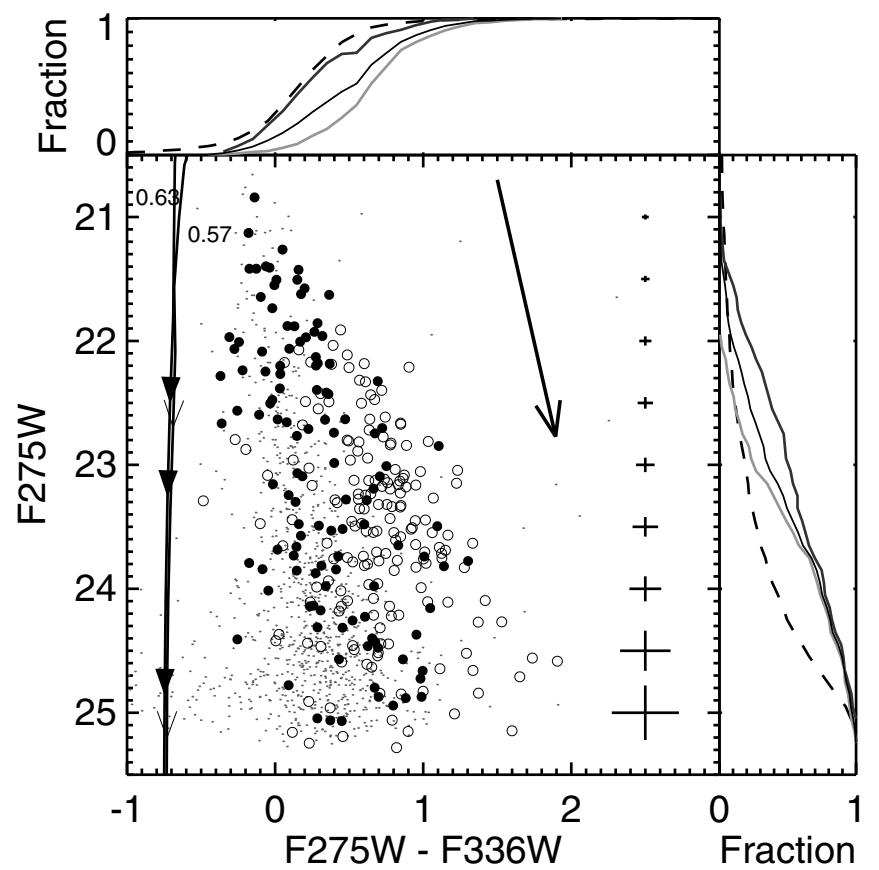

Figure 16. Filled black circles denote low-EC PNs. Open circles denote medium- to high-EC PNs. Background gray dots indicate stars within $3^{\prime \prime}$ of a PN. Crosses along the left side of the plot indicate median Poisson errors from the photometry of PNs binned by F275W. A reddening vector shows the extinction effect of $A_{V}=1$. Also shown are two unreddened P-AGB tracks for initial masses of $0.57 M_{\odot}$ and $0.63 M_{\odot}$. The tracks are nearly identical in this region of color-magnitude space. Open arrows indicate $0.57 M_{\odot} 20,000$ and $30,000 \mathrm{yr}$ ages. Filled arrows indicate $0.63 M_{\odot} 2000,3000$, and 4000 ages. Top: the normalized cumulative distribution of F275W-F336W for PNs (thin black line), low-EC PNs (dark gray line), medium- to high-EC PNs (light gray line), and stars within $3^{\prime \prime}$ of a PN (dashed line). Right: the normalized cumulative distribution of F275W for PNs (thin black line), low-EC PNs (dark gray line), medium- to high-EC PNs (light gray line), and stars within $3^{\prime \prime}$ of a PN (dashed line). Low-EC PNs tend to be brighter and bluer than medium- to high-EC PNs in the PHAT UV CMD.

$\mathrm{F} 110 \mathrm{~W}-\mathrm{F} 160 \mathrm{~W}=0$, with only younger MS stars $(<100 \mathrm{Myr})$ expected to have bluer colors at F110W $\lesssim 23$. The lack of young MS stars neighboring PNs (Figure 11) emphasizes that PNs are not sampling regions with very recent star formation.

\subsection{Spectral Energy Distributions}

For PNs with detections in all six PHAT filters, converting from magnitude to total flux produces SEDs covering $0.3-1.6 \mu \mathrm{m}$. Such SEDs will provide constraints for future models of PNs evolution. Such models will be necessary to reliably constrain extinction for these PNs. Figure 18 shows SEDs for 130 PNs with detections in all six filters.

The right column of Figure 18 shows SEDs grouped by $m 5007$. SEDs of the brightest PNs are largely uniform while SEDs of fainter PNs show more variations. The SEDs look much like those of hot stars with the addition of a prominent peaks at 0.475 and $1.1 \mu \mathrm{m}$ due to emission lines in the bandpasses. However, unlike hot stars, flux at $0.336 \mu \mathrm{m}$ exceeds flux at $0.275 \mu \mathrm{m}$ for many PNs. This could be due to the fact that $0.275 \mu \mathrm{m}$ flux is more attenuated by extinction, combined with the larger uncertainties in $0.275 \mu \mathrm{m}$ flux.

The left column of Figure 18 shows SEDs grouped by EC. At the lowest EC, the SEDs show little evidence of emission-line flux above the blue stellar continuum. Moving through higher $\mathrm{EC}$, the emission-line features become much more prominent. Again, this suggests low-EC PNs are largely young PNs, with

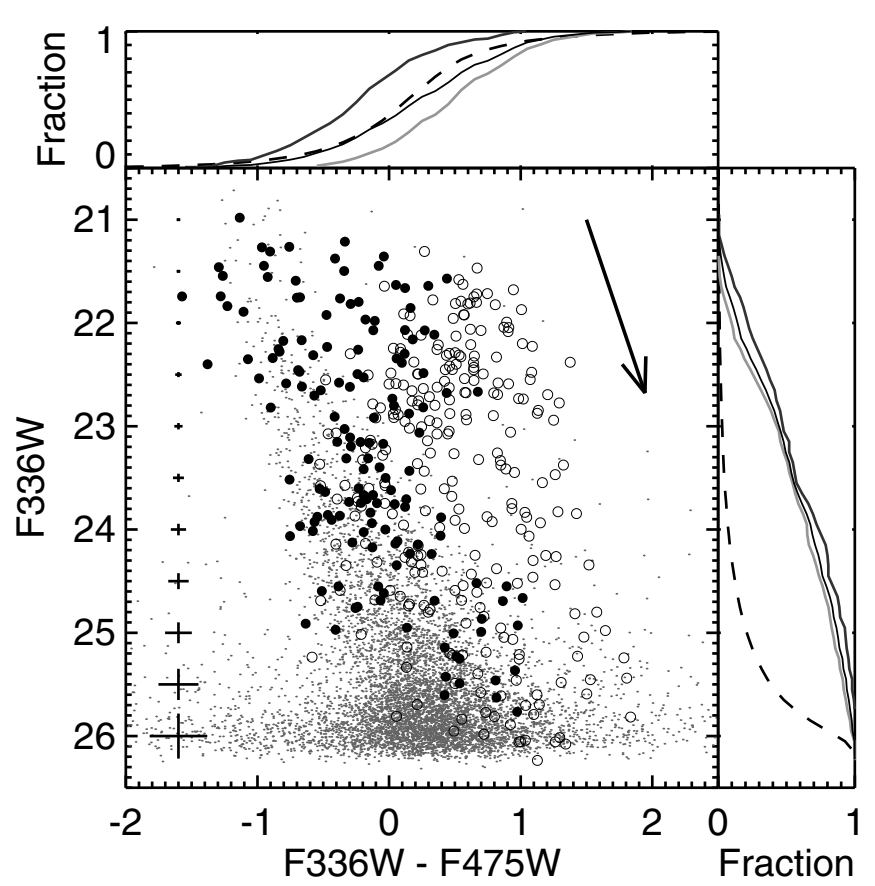

Figure 17. Filled black circles denote low-EC PNs. Open circles denote medium- to high-EC PNs. Background gray dots indicate stars within $3^{\prime \prime}$ of a PN. Crosses along the left side of the plot indicate median Poisson errors from the photometry of PNs binned by F336W. A reddening vector shows the extinction effect of $A_{V}=1$. Top: the normalized cumulative distribution of F336W-F475W for PNs (thin black line), low-EC PNs (dark gray line), medium- to high-EC PNs (light gray line), and stars within $3^{\prime \prime}$ of a PN (dashed line). Right: the normalized cumulative distribution of F336W for PNs (thin black line), low-EC PNs (dark gray line), medium- to high-EC PNs (light gray line), and stars within $3^{\prime \prime}$ of a PN (dashed line). A separation in excitation becomes apparent when comparing emission flux to continuum flux.

the lowest-EC PNs being just beyond the emergence of [O III] lines, which grow stronger as PNs evolve to higher EC.

The two anomalously NIR-bright PNs also show up in the SEDs. Again, it is likely they are crowded by a NIRbright source.

\subsection{Resolved PNs and Size Estimates}

PNs are commonly found to have radii on the order of $0.1 \mathrm{pc}$, but have been known to have radii as large as $0.6 \mathrm{pc}$ (Phillips 2003). It might be expected that PNs with radii $>0.5$ pc could be resolved in PHAT considering the HST ACS camera has a plate scale of $0^{\prime \prime} .05$ pixel $^{-1}\left(0.2 \mathrm{pc}\right.$ pixel $^{-1}$ at the distance of M31) and the average FWHM of PHAT's PSF is $\sim 2$ pixels. However, as PNs evolve, they both expand radially and drop in luminosity, resulting in a steep decline of surface brightness. PNs large enough to be resolved in PHAT are likely too faint to be detected in PHAT. At intermediate stages of evolution, however, some PNs may be bright enough to be detected in PHAT and large enough to be slightly resolved. Figure 19 shows the dependence of FWHM on magnitude in PHAT data. Faint sources in crowded regions suffer heavy blending that can cause the direct FWHM measurement to overestimate the true FWHM. However, the FWHM distribution of PNs is indistinguishable from that of nearby stars, suggesting that PNs are not resolved in PHAT.

In general, the PNs sample does not appear any more extended than nearby stars; however, we found one PN (M06 143) that appeared significantly larger than the surrounding stars. We show an image of this PN in Figure 20. This PN has a FWHM of $\sim 4$ pixels, compared to stars in the area, which have FWHMs of 

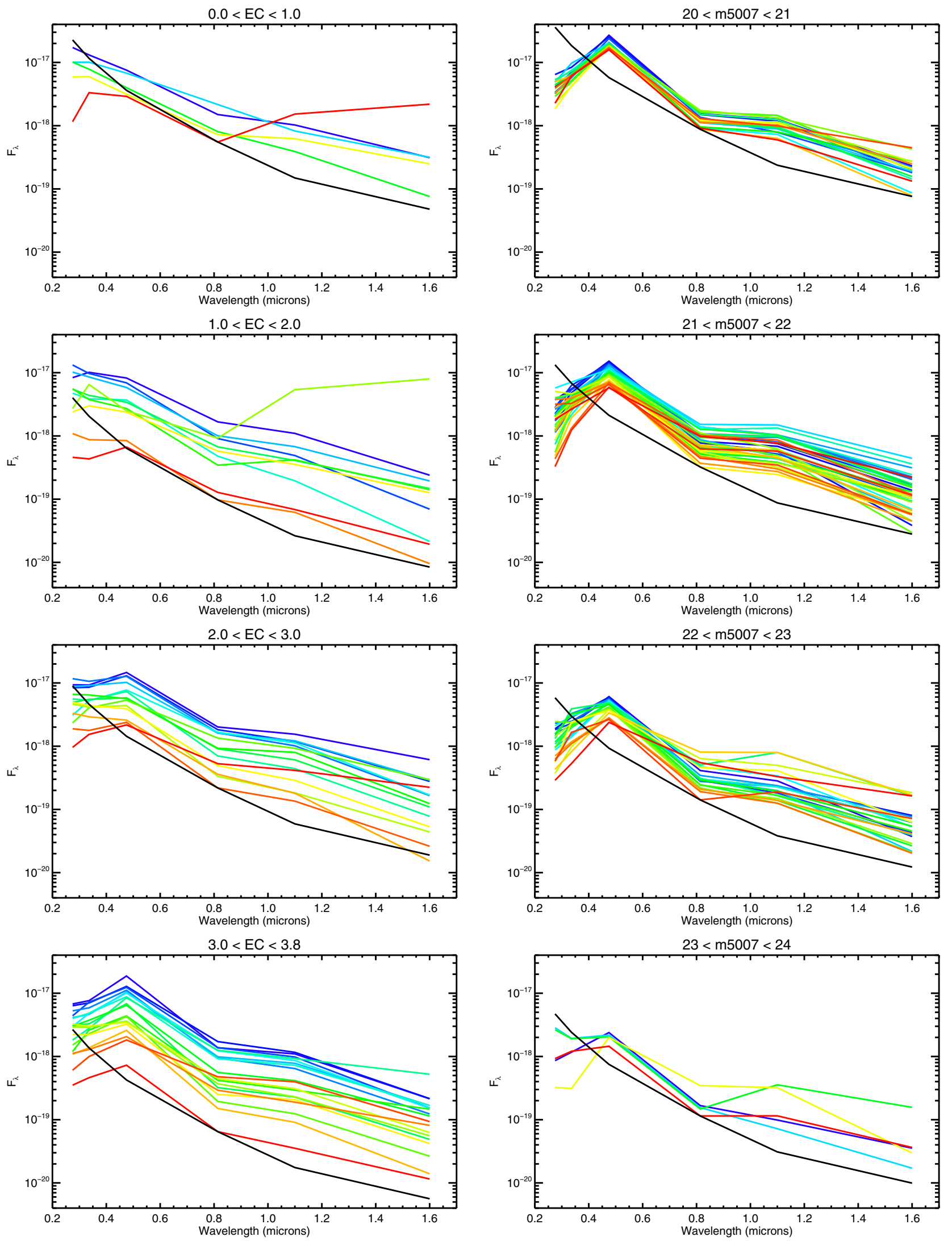

Figure 18. Spectral energy distributions of PHAT PNs with detections in all six bandpasses. The left column of plots shows SEDs of low-EC PNs sorted by EC. The right column of plots show SEDs of medium- to high-EC PNs sorted by $m 5007$. All SED plots are color-ranked by relative F475W flux. Black lines denote a $50,000 \mathrm{~K}$ blackbody curve accounting for full system throughput and extinction-corrected for $A_{V}=0.17\left(R_{V}=3.1\right)$, the average foreground extinction for the PHAT region (Schlafly \& Finkbeiner 2011). Increasing excitation correlates to increasing emission-line flux over the underlying continuum in the F475W and F110W bandpasses. (A color version of this figure is available in the online journal.) 


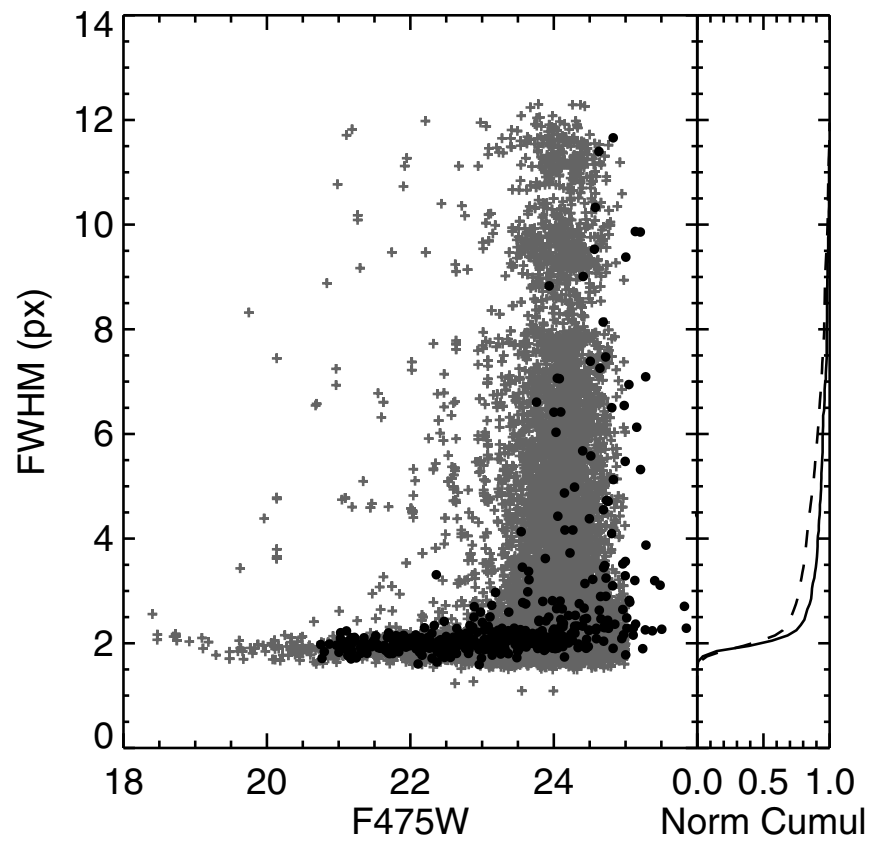

Figure 19. Left: direct FWHM measurements of PNs (black circles) and the 50 brightest stars with $\mathrm{F} 475 \mathrm{~W}<25$ around each $\mathrm{PN}$ (gray pluses). FWHM values greater than $\sim 3$ are likely due to poor direct FWHM measurement of faint, crowded sources. Right: normalized cumulative histograms of FWHM for PNs (solid line) and stars (dashed line). The PHAT PNs catalog does not appear extended compared to neighboring stars.

$\sim 2$ pixels. After accounting for the effects of a $0^{\prime \prime} 1$ FWHM PSF, the intrinsic radius of the PN would be up to 0 '. 08 , or $0.3-0.4 \mathrm{pc}$. The PHAT magnitudes of M06 143 are likely underestimated due to the extended nature of the PSF. This is supported by the fact that M06 143 is the faintest PN in our sample, with $\mathrm{F} 475 \mathrm{~W}=26.15$, and has $\mathrm{F} 475 \mathrm{~W}-m 5007=1.4$ - the largest difference of the PNs sample and more than three times the F475W $-m 5007$ saturation limit of the PNs sample. Though the optical color of M06 143 is consistent with PNs in PHAT, we cannot rule out the possibility it is a blended source or a non-PN source.

Finally, any M06 source that was visually resolved in PHAT was rejected as being a PN (M06 143 is the only marginally extended PN) and classified as a possible $\mathrm{H}$ II region. Therefore, inclusion in Table 3 serves as an updated classification of extended objects in M06, which will be more reliable than such classifications from the ground-based data.

\section{SUMMARY}

We have identified 467 PNs in the PHAT survey photometry catalogs. The $\mathrm{F} 475 \mathrm{~W}$ broadband magnitudes show a tight correlation with the $m 5007$ magnitudes clearly indicating the purity of our sample. These PNs are unresolved and dominated by [O III] line emission. Since the PHAT astrometric solution is precise to 0 .'01, we have provided improved astrometry for all of the PNs in our sample, along with six-band photometry from the PHAT survey.

Comparing our broadband magnitudes and M06 narrowband magnitudes to ECs from optical spectroscopy in the literature shows that low-excitation PNs tend to have bluer colors in F475W-m5007, suggesting that our broadband magnitudes in these cases probe the emission from the central star. These

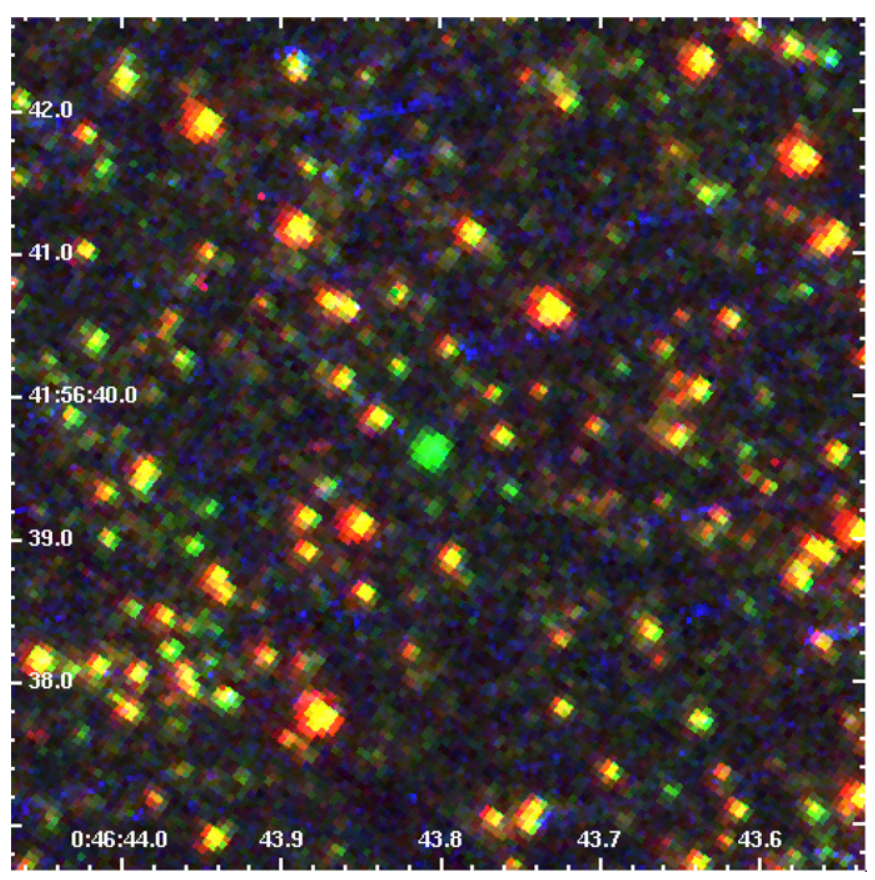

Figure 20. Bright green object in the center of the frame is M06 143-a slightly resolved $\mathrm{PN}$ candidate.

(A color version of this figure is available in the online journal.)

results indicate that our SEDs will be useful for constraining models of PNs and central star evolution.

The size distribution of PNs in our HST images are consistent with that of point sources. However, there is some evidence that at least one PN is marginally resolved, corresponding to a physical radius of up to $0.3-0.4 \mathrm{pc}$.

Support for this work was provided by NASA through grant GO-12055 from the Space Telescope Science Institute, which is operated by the Association of Universities for Research in Astronomy, Incorporated, under NASA contract NAS5-26555.

\section{REFERENCES}

Bianchi, L., Vassiliadis, E., \& Dopita, M. 1997, ApJ, 480, 290 Ciardullo, R., Jacoby, G. H., Ford, H. C., \& Neill, J. D. 1989, ApJ, 339, 53 Dalcanton, J. J., Fouesneau, M., Hogg, D. W., et al. 2014, ApJ, submitted Dalcanton, J. J., Williams, B. F., Lang, D., et al. 2012, ApJS, 200, 18 Dolphin, A. E. 2000, PASP, 112, 1383

Dopita, M. A., \& Meatheringham, S. J. 1990, ApJ, 357, 140 Dopita, M. A., \& Meatheringham, S. J. 1991a, ApJ, 367, 115 Dopita, M. A., \& Meatheringham, S. J. 1991b, ApJ, 377, 480 Dopita, M. A., Meatheringham, S. J., Webster, B. L., \& Ford, H. C. 1988, ApJ, 327,639

Dopita, M. A., Meatheringham, S. J., Wood, P. R., et al. 1987, ApJL, 315, L107 Douglas, N. G., Arnaboldi, M., Freeman, K. C., et al. 2002, PASP, 114, 1234 Draine, B. T., Aniano, G., Krause, O., et al. 2014, ApJ, 780, 172

Ferland, G. J., Porter, R. L., van Hoof, P. A. M., et al. 2013, RMxAA, 49, 137

Girardi, L., Dalcanton, J., Williams, B., et al. 2008, PASP, 120, 583

Gurzadyan, G. A., \& Egikian, A. G. 1991, Ap\&SS, 181, 73

Halliday, C., Carter, D., Bridges, T. J., et al. 2006, MNRAS, 369, 97

Hurley-Keller, D., Morrison, H. L., Harding, P., \& Jacoby, G. H. 2004, ApJ, 616,804

Kniazev, A. Y., Grebel, E. K., Zucker, D. B., et al. 2014, AJ, 147, 16

Kwitter, K. B., Lehman, E. M. M., Balick, B., \& Henry, R. B. C. 2012, ApJ, 753,12

Kwok, S. 2000, The Origin and Evolution of Planetary Nebulae (Cambridge: Cambridge Univ. Press) 
Marigo, P., Girardi, L., Groenewegen, M. A. T., \& Weiss, A. 2001, A\&A, 378, 958

Marigo, P., Girardi, L., Weiss, A., Groenewegen, M. A. T., \& Chiosi, C. 2004, A\&A, 423, 995

Massey, P., Olsen, K. A. G., Hodge, P. W., et al. 2006, AJ, 131, 2478

McConnachie, A. W., Irwin, M. J., Ferguson, A. M. N., et al. 2005, MNRAS, 356, 979

Méndez, R. H., Teodorescu, A. M., Schönberner, D., Jacob, R., \& Steffen, M. 2008, ApJ, 681, 325

Merrett, H. R., Merrifield, M. R., Douglas, N. G., et al. 2006, MNRAS, 369, 120
Perinotto, M., Schönberner, D., Steffen, M., \& Calonaci, C. 2004, A\&A, 414, 993

Phillips, J. P. 2003, AN, 324, 191

Reid, W. A., \& Parker, Q. A. 2010, PASA, 27, 187

Sanders, N. E., Caldwell, N., McDowell, J., \& Harding, P. 2012, ApJ, 758,133

Schlafly, E. F., \& Finkbeiner, D. P. 2011, ApJ, 737, 103

Schönberner, D., Jacob, R., Steffen, M., \& Sandin, C. 2007, A\&A, 473, 467

Thilker, D. A., Hoopes, C. G., Bianchi, L., et al. 2005, ApJL, 619, L67

Vassiliadis, E., \& Wood, P. R. 1993, ApJ, 413, 641

Williams, B. F., Lang, D., Dalcanton, J. J., et al. 2014, ApJS, submitted 